\title{
GRAPHS WITH THE CIRCUIT COVER PROPERTY
}

\author{
BRIAN ALSPACH, LUIS GODDYN, AND CUN-QUAN ZHANG
}

\begin{abstract}
A circuit cover of an edge-weighted graph $(G, p)$ is a multiset of circuits in $G$ such that every edge $e$ is contained in exactly $p(e)$ circuits in the multiset. A nonnegative integer valued weight vector $p$ is admissible if the total weight of any edge-cut is even, and no edge has more than half the total weight of any edge-cut containing it. A graph $G$ has the circuit cover property if $(G, p)$ has a circuit cover for every admissible weight vector $p$. We prove that a graph has the circuit cover property if and only if it contains no subgraph homeomorphic to Petersen's graph. In particular, every 2-edge-connected graph with no subgraph homeomorphic to Petersen's graph has a cycle double cover.
\end{abstract}

\section{INTRODUCTION}

Let $(G, p)$ be an edge-weighted graph (with loops and multiple edges allowed) where $p: E(G) \rightarrow \mathbb{Z}$. The following question, which we shall call the circuit cover problem, has attracted considerable interest since it was posed and solved for planar graphs by P. D. Seymour in 1979 [Sey1]: "Find conditions on $(G, p)$ for there to exist a multiset (or list) $\mathbf{L}$ of circuits in $G$ such that each edge $e$ is 'covered' exactly $p(e)$ times by circuits in L." More precisely, we say that $(G, p)$ has a circuit cover (or that $G$ has a circuit $p$-cover) provided the following holds:

(1.1) There exists a vector of nonnegative integer coefficients $\left(\lambda_{C}: C \in \mathbf{C}\right)$ such that $\sum_{C \in \mathrm{C}} \lambda_{C} \chi^{C}=p$.

(Here, C denotes the collection of circuits in $G$, and $\left(\lambda_{C}\right)$ is the multiplicity vector for the circuit cover $\mathrm{L}$, and for any subgraph $H$ of $G, \chi^{H}$ denotes the $\{0,1\}$-characteristic vector of the edge set of $H$.)

The circuit cover problem is related to problems involving graph embeddings [Arc, Hag, Lit, Tut], flow theory [Cel, Fan2, Jae1, You], short circuit covers [Alo, Ber, Fan1, Gua, Jac, Jam2, Jam3, Tar1, Zha1], the Chinese Postman Problem [Edm, Gua, Ita, Jac], perfect matchings [Ful, God2, p. 22] and decompositions of eulerian graphs [Fle1, Fle2, Sey3]. When $p$ is the constant vector 1 (or any odd number), we are characterizing eulerian graphs. When $p=2$ we have the well known cycle double cover conjecture. The cases $p=4$ and $p=6$ have been settled for graphs using the 8- and 6-flow theorems by Jaeger [Jae1] and

Received by the editors June 4, 1990 and, in revised form, April 1, 1992 and September 9, 1992. 1991 Mathematics Subject Classification. Primary 05C38; Secondary 05 C70.

The first author was partially funded by Natural Sciences and Engineering Research Council grant \# A-4792. The second author was partially funded by Natural Sciences and Engineering Research Council grant \# A-5499. The third author was partially funded by National Science Foundation grant \# DMS-8906973. 
Fan [Fan2] respectively. Tarsi [Tar1] generalizes the case where $p$ is constant to the class of binary matroids.

Seymour [Sey1] gave three necessary conditions for an arbitrary weighted graph $(G, p)$ to have a circuit cover:

(i) $p$ is nonnegative integer valued;

(ii) for every edge-cut $B$ and $e \in B, p(e) \leq p(B \backslash e)$, (that is, $p$ is balanced);

(iii) for every edge-cut $B, p(B)$ is even, (that is, $p$ is eulerian).

(We use the convention that $p(F)$ means $\sum_{e \in F} p(e)$, for any $F \subseteq E$.) These conditions follow easily from the observation that any circuit in a graph intersects any edge-cut in an even number of edges. The conditions in (1.2) are collectively called admissibility conditions, and $p$ is said to be admissible if it satisfies (1.2).

Our main result characterizes the graphs for which (1.1) and (1.2) are equivalent. We say that a graph $G$ has the circuit cover property if $(G, p)$ has a circuit cover for every admissible weight $p$.

Not every graph has the circuit cover property. Let $P_{10}$ denote Petersen's graph and let $p_{10}$ take the value 1 on some 2-factor of $P_{10}$, and the value 2 on the complementary 1 -factor. Then $\left(P_{10}, p_{10}\right)$ is admissible, but has no circuit cover, as has been observed by several authors [Sey1, Sze, Zel]. Clearly, no graph homeomorphic to $P_{10}$ has the circuit cover property. By assigning weight zero to deleted edges, it is easy to see that no graph which has a $P_{10}$-minor (a minor isomorphic to $P_{10}$ ) has the circuit cover property. (Since $P_{10}$ is cubic, a graph has a $P_{10}$-minor if and only if some subgraph of $G$ is homeomorphic to $P_{10}$.)

The pertinence of $P_{10}$ to the circuit cover problem for cubic graphs was established by Alspach and Zhang [Als] where they showed that a cubic graph $G$ has a circuit $p$-cover for every admissible $\{0,1,2\}$-valued weight vector $p$ if and only if $G$ has no $P_{10}$-minor. Our main result generalizes this result to arbitrary weighted graphs.

Theorem 1. A graph has the circuit cover property if and only if it has no $P_{10^{-}}$ minor.

We give some terminology: In this paper, graphs are finite, undirected with loops and multiple edges allowed. Because of a strong connection to matroid theory, we borrow some terms from that area (see [Wel] for an introduction to matroid theory). Most notably, a cycle (or even subgraph) in a graph $G=$ $(V, E)$ is a subset of edges $F \subseteq E$ such that each vertex of $G$ is incident with an even number of edges in $F$. A circuit is a minimal nonempty cycle. Since any cycle is an edge-disjoint union of circuits, $(G, p)$ has a cycle cover if and only if it has a circuit cover. Where no confusion arises, we identify a cycle with the subgraph of $G$ induced by the cycle. For example, we use $V(C)$ to denote the set of vertices of a circuit $C$. A graph is eulerian if it is connected and its edge set forms a cycle. If $e \in E(G)$ then $G \backslash e$ denotes the graph obtained from $G$ by deleting $e$, and $G / e$ denotes the graph obtained from $G$ by contracting $e$ (that is, we identify the endvertices of $e$, then delete $e$ ). Loops and multiple edges (other than $e$ ) which arise from a contraction are not deleted. Any graph obtained from $G$ by successive deletions and contractions is called a minor of $G$. The order in which edges are deleted and contracted is irrelevant, so any 
minor of $G$ may be written as $G \backslash E_{1} / E_{2}$ where $E_{1}$ and $E_{2}$ are disjoint subsets of $E(G)$. If $H$ is a cubic graph then $H$ is a minor of $G$ if and only if some subgraph of $G$ is homeomorphic to $H$. For any subset $S$ of vertices of $G$, the set of edges $\delta(S)=[S, V-S]$ which have exactly one endvertex in $S$ is called an edge-cut (or cocycle) of $G$. A bond (or cocircuit) is a minimal nonempty edge-cut. A bridge (or coloop) is an edge-cut of cardinality 1. A graph with no bridges is said to be bridgeless.

There are several consequences of Theorem 1 . Since $P_{10}$ is nonplanar, we obtain the following classic result of P. D. Seymour.

Corollary 1 [Sey1]. Every planar graph has the circuit cover property.

Since $P_{10}-v$ is nonplanar for any vertex $v$ we have the following sharpening.

Corollary 2. If $G-v$ is planar for some vertex $v$, then $G$ has the circuit cover property.

The well-known cycle double cover conjecture asserts that every bridgeless graph has a circuit 2-cover. This conjecture has been the subject of numerous papers [Bon, Cat, God1, God2, Jae2, Sey1, Sze, Tar2], and has been verified for various classes of graphs. The following corollary is new, although it was previously known to hold for cubic graphs [Als].

Corollary 3. Every bridgeless graph with no $P_{10}$-minor has a cycle double cover.

A graph $G$ is said to have a nowhere zero 4-flow if $E(G)=E_{1} \cup E_{2}$ where each $E_{i}$ is a cycle in $G$ [Jael, Mat]. Every graph with a nowhere zero 4-flow has a cycle double cover, namely $\left\{E_{1}, E_{2}, E_{1} \triangle E_{2}\right\}$. Corollary 3 thus lends support to the following well-known conjecture of Tutte.

Conjecture 1 [Tut]. Every bridgeless graph with no $P_{10}$-minor has a nowhere zero 4-flow.

Several authors have investigated a relationship between the Chinese Postman Problem and the Shortest Circuit Cover Problem. Let $G$ be a graph. Using our notation, the Chinese Postman Problem [Ber, Edm, Gua, Ita, Jac] essentially is to find the smallest integer $c_{G}$ such that there exists an eulerian weight vector $p \geq 1$ satisfying $p(G)=c_{G}$. The Shortest Circuit Cover Problem [Ber, Fan, Gua, Jac, Jam2, Tar1] is to find the smallest integer $s_{G}$ such that $(G, p)$ has a circuit cover for some (admissible) weight vector $p \geq 1$ satisfying $p(G)=s_{G}$ ( $s_{G}$ is not defined if $G$ has a bridge). It is immediate from the definitions that for any bridgeless graph $G$,

$$
c_{G} \leq s_{G}
$$

In general we do not have equality since $c_{P_{10}}=20$ while $s_{P_{10}}=21$ (see [Ita]). For bridgeless graphs we have the general upper bounds $c_{G} \leq 4|E(G)| / 3$ and $s_{G} \leq 5|E(G)| / 3$ [Ber], though it is conjectured that $s_{G} \leq 7|E(G)| / 5$. (Jamshy and Tarsi [Jam3] have shown that this last inequality actually implies the cycle double cover conjecture.)

Although there is a polynomial-time algorithm for determining $c_{G}$ [Edm], the determination of $s_{G}$ is considered to be a very difficult problem [Ber, Gua, Jam2, Tar1]. Hence there is considerable interest in determining classes of graphs for which equality holds in (1.3). It is known [Gua, Ber] that equality 
holds for all bridgeless planar graphs. This class was extended by Alspach and Zhang [Als] to include all bridgeless cubic graphs which have no $P_{10}$-minor. Both of these results follow from the fact that equality holds in (1.3) for any bridgeless graph $G$ which has the circuit cover property. (This fact follows easily from Proposition 6 and by observing [Edm] that any eulerian vector $p \geq 1$ with $p(G)=c_{G}$ is $\{1,2\}$-valued.) From Theorem 1 we have the following generalization.

Corollary 4. If $G$ is a bridgeless graph with no $P_{10}$-minor, then $s_{G}=c_{G}$.

It is known [Ber, Jac, Zha1] that $s_{G}=c_{G}$ whenever $G$ has a nowhere zero 4-flow. This fact, together with Corollary 4, indirectly lends further support to Conjecture 1.

We compare Theorem 1 to a theorem of Fleischner and Frank [Fle2] regarding decompositions of eulerian graphs into circuits which avoid certain "forbidden" sets of edges. For each vertex $v$ of an eulerian graph $G$ a partition $P(v)$ of the edges incident with $v$ is specified. We set $P=\bigcup_{v \in V(G)} P(v)$ and call each member of $P$ a forbidden part. A decomposition of $E(G)$ into circuits is good (with respect to $P$ ) if no circuit contains two edges from a single forbidden part. The problem is to establish conditions on $(G, P)$ under which there exists a good decomposition of $G$ with respect to $P$.

Theorem 2 [Fle2]. A planar eulerian graph has a good decomposition with respect to $P$ if and only if no edge-cut $B$ contains more than $|B| / 2$ edges belonging to the same forbidden set.

Suppose $(G, p)$ is a planar graph with an admissible edge-weight vector. Let $H$ be the planar eulerian graph obtained from $G$ by replacing each $e \in$ $E(G)$ with $p(e)$ parallel edges. Let these sets of parallel edges constitute a collection $P$ of forbidden parts for $H$. Since $(G, p)$ is balanced, the pair $(H, P)$ satisfies the hypothesis of Theorem 2 . One easily sees that a good decomposition of $H$ with respect to $P$ corresponds to a circuit cover of $(G, p)$. It follows that Theorem 2 implies Corollary 1 . Theorem 2 appears to be a strict generalization of Corollary 1 since there does not seem to be a reverse transformation $(H, P) \rightarrow(G, p)$ which preserves planarity. Theorems 1 and 2 appear to generalize Corollary 1 in very different ways since it is not at all clear that Corollaries 2 or 3 can be derived from Theorem 2. Seymour [Sey3] uses Corollary 1 to prove his Even Circuit Decomposition Theorem. One of the present authors [Zha2, Zha3] has used a strong form of the main theorem of this paper (see Theorem 4) to generalize both Theorem 2 and Seymour's Even Circuit Decomposition Theorem to the class of graphs with no $K_{5}$-minor.

A further consequence of Theorem 1 involves the natural generalization of circuit covers to weighted matroids $(M, p)$. For binary matroids, the conditions in (1.2) are still necessary for $(M, p)$ to have a circuit cover, where "edge-cut" is replaced by "cocircuit". The penultimate conjecture in [Sey2] proposes a forbidden minor characterization of binary matroids with the circuit cover property:

Conjecture 2 [Sey2]. A binary matroid $M$ has the circuit cover property if and only if no minor of $M$ is isomorphic to either $M\left(P_{10}\right), M^{*}\left(K_{5}\right), F_{7}^{*}$, or $R_{10}$.

(See [Sey2] for definitions.) By using Theorem 1 together with Seymour's 
matroid decomposition theorems, (see [Sey2]), Fu and Goddyn have settled this conjecture affirmatively [Fu].

The relaxation of (1.1) to nonnegative rational coefficients has been studied for both graphs [Sey1] and matroids [Sey2]. A weight vector $p$ is in the cone of circuits of $M$ if there is a nonnegative rational vector $\left(\alpha_{C}\right)_{C \in \mathbf{C}}$ satisfying $\sum_{C \in \mathrm{C}} \alpha_{C} \chi^{C}=p$. There are two natural necessary conditions for a vector $p$ to be in the cone of circuits of a matroid; $p$ must be nonnegative and balanced. We say that a matroid $M$ has the sums of circuits property if every balanced nonnegative rational weight vector $p$ is in the cone of circuits of $M$. A forbidden-minor characterization of those matroids with the sums of circuits property is given by Seymour.

Theorem 3 [Sey2]. A matroid $M$ has the sums of circuits property if and only if $M$ is binary and no minor of $M$ is isomorphic to either $M^{*}\left(K_{5}\right), F_{7}^{*}$ or $R_{10}$.

In particular, every graph has the sums of circuits property. We note that this list of forbidden minors is the same as that for Conjecture 2 except for Petersen's graph. Conjecture 2 might be considered to be an "integer analog" of Theorem 3.

\section{A STRONGER THEOREM}

We shall prove something slightly stronger than Theorem 1 . This stronger version (Theorem 4 below) is needed for some applications of Zhang [Zha2, Zha3]. We say that a weighted graph $(G, p)$ is contra-weighted if $(G, p)$ is admissible, but has no circuit cover.

First we note three trivial operations that can yield contra-weighted graphs other than $\left(P_{10}, p_{10}\right)$ :

(i) new vertices and edges of weight zero may be added,

(ii) any edge may be subdivided into a path of edges of the same weight,

(iii) if some vertex of degree 2 is adjacent to two edges of weight 2 , then one of these edges may be replaced by two parallel edges of weight 1 .

Any weighted graph obtainable from $\left(P_{10}, p_{10}\right)$ by repeated application of these three operations is called a blistered $P_{10}$. A typical blistered $P_{10}$ appears in Figure 1 (edges of weight 0 are not shown). Note that $\left(P_{10}, p_{10}\right)$ is the only 3-connected blistered $P_{10}$ which has no edges of weight 0 .

We write $p \leq q$, if $p(e) \leq q(e)$ for all $e \in E$.

Theorem 4. If $(G, p)$ is a contra-weighted graph, then there exists $q \leq p$ such that $(G, q)$ is a blistered $P_{10}$.

Theorem 4 follows from two lemmas whose proofs comprise the next three sections of this paper.

Lemma 1. If $(G, p)$ is a contra-weighted graph, then there exists $q \leq p$ such that $(G, q)$ is a $\{0,1,2\}$-valued contra-weighted graph. 


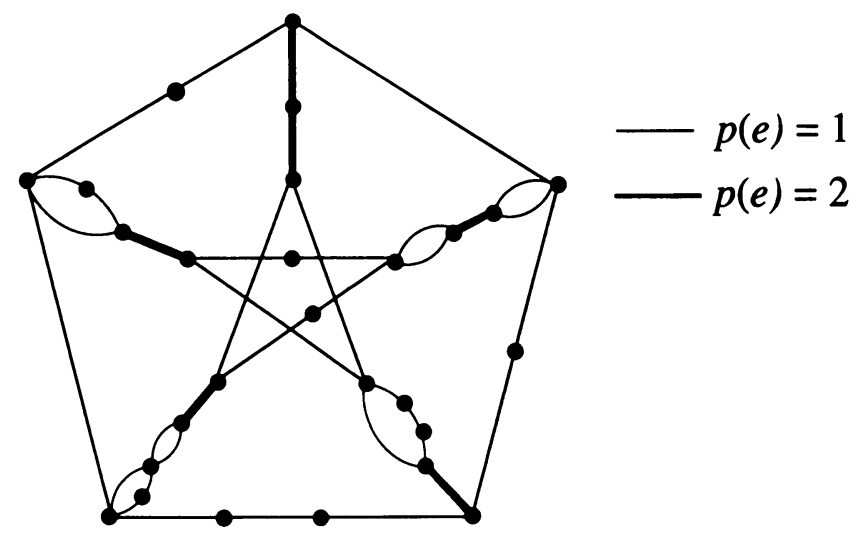

FIGURE 1

Lemma 2. If $(G, p)$ is a $\{1,2\}$-valued contra-weighted graph, then there exists $q \leq p$ such that $(G, q)$ is a blistered $P_{10}$.

As we shall see, the proof of Lemma 1 has the flavour of Seymour's proof of Corollary 1, while the proof of Lemma 2 is essentially an extension of that given by Alspach and Zhang in [Als].

\section{Preparation for Lemma 1}

We say that $p$ is positive if $p \geq \mathbf{1}$. We lose no generality when we assume $p$ is positive since edges of weight zero simply may be deleted.

We first study special edge-cuts. Let $(G, p)$ be a positive, balanced weighted graph. An edge-cut $B$ is called a tight cut if $p(e)=p(B \backslash e)$ for some $e \in B$. In this case, $e$ is called a tight cut leader for $B$, and any other edge in $B$ is called a tight cut follower for $B$. Since $p$ is positive and balanced, any tight cut must be a bond. Furthermore, $B$ has a unique tight cut leader provided $|B| \geq 3$. If $|B|=2$, then each edge in $B$ is both a leader and a follower. The importance of tight cuts is manifested in the following observation of Seymour [Sey1].

Proposition 1. In any circuit cover of $(G, p)$, every circuit which intersects with a tight cut $B$ contains a tight cut leader for $B$, and exactly one other edge in $B$.

Let $e, f \in E(G)$. We say that $e$ follows $f$ in $(G, p)$ if either $e=f$ or some tight cut $B$ has $e$ as a follower and $f$ as a leader.

Proposition 2. If $p$ is balanced, then "follows" is a transitive relation on $E(G)$.

Proof. Let $B$ be a tight cut in which $e$ follows $f$ and let $C$ be a tight cut in which $f$ follows $g$. If $e=g$, then $B=C$ and $|B|=2$ follows from the paragraph preceding Proposition 1 , in which case Proposition 2 holds. Thus we assume $e \neq g$. By definition, $f \in B \cap C$. If either $e$ or $g$ is in $B \cap C$, then we are done since both $B$ and $C$ are tight. Hence we assume that $e \in B \backslash C$, $f \in B \cap C$, and $g \in C \backslash B$. Thus $e, g \in B \triangle C$. We have the following sequence 
of inequalities:

$$
\begin{array}{rlrl}
2 p(g) & =p(C \backslash B)+p(C \cap B) & & \text { (since } C \text { is tight) } \\
& \geq p(C \backslash B)+p(f) & & \text { (since } f \in B \cap C) \\
& =p(C \backslash B)+p(B \backslash f) & & \text { (since } B \text { is tight) } \\
& \geq p(C \backslash B)+p(B \backslash C) & & \text { (since } f \in C) \\
& \geq 2 p(g) & & \text { (since } B \triangle C \text { is an edge-cut, and hence } \\
& & \text { is balanced). }
\end{array}
$$

We have equality throughout. In particular, $B \triangle C$ is a tight cut in which $2 p(g)=p(B \triangle C)$ and therefore $e$ follows $g$.

For any collection $\mathbf{H}=\left\{H_{1}, \ldots, H_{m}\right\}$ of subgraphs of $G$, denote by $\chi^{\mathbf{H}}$ the sum of the characteristic vectors $\chi^{H_{i}}, i=1,2, \ldots, m$.

Proposition 3. If $\mathbf{H}$ is a collection of circuits in $G$, then $\left(G, \chi^{\mathbf{H}}\right)$ is admissible.

Proposition 4. Let $F \subseteq E(G)$ and let $p_{F}$ denote the restriction of $p$ to $E(G) \backslash F$. If $(G, p)$ has a circuit cover, then so does the contracted graph $\left(G / F, p_{F}\right)$.

A minimal contra-weighted graph is a contra-weighted graph $(G, p)$ such that $(G, q)$ is not contra-weighted for any $q<p$. Although it is possible that minimal contra-weighted graphs have tight cuts, such tight cuts must be "well behaved".

Proposition 5. Let $(G, p)$ be a positive minimal contra-weighted graph and let $B$ be a tight cut in $(G, p)$ with a leader $e_{1} \in B$. Then there exists a sequence $X=\left(x_{1}, x_{2}, \ldots, x_{k}\right)$ of distinct vertices such that $B=\delta(X)$ and, for $i=$ $1,2, \ldots, k, \delta\left(\left\{x_{1}, x_{2}, \ldots, x_{i}\right\}\right)$ is a tight cut having $e_{1}$ as a leader.

Proof. In a minimal contra-weighted graph $(G, p)$, let $B=\left[X_{1}, X_{2}\right]$ be a tight cut with a leader $e_{1}=x_{1} y_{1}, x_{1} \in X_{1}, y_{1} \in X_{2}$. Let $\left(G_{i}, p_{i}\right)$ be the weighted graph obtained by contracting the edges with neither endvertex in $X_{i}, i=1,2$. That is, $G_{i}=G / E\left(G\left[X_{3-i}\right]\right)$.

We claim that either $\left(G_{1}, p_{1}\right)$ or $\left(G_{2}, p_{2}\right)$ is contra-weighted. Since edge contraction introduces no new edges or edge-cuts, each $\left(G_{i}, p_{i}\right)$ is positive and admissible, just as $(G, p)$ is. If neither $\left(G_{1}, p_{1}\right)$ nor $\left(G_{2}, p_{2}\right)$ were contraweighted, then they would both have circuit covers. By Proposition 1, we could then pair off the circuits which contain $e_{1}$ in $G_{1}$ with those in $G_{2}$, obtaining a circuit cover of $(G, p)$. This establishes the claim.

Assume that $\left(G_{2}, p_{2}\right)$ is contra-weighted. Let $e_{1}=x_{1} y_{2} \in E\left(G_{1}\right)$, where $x_{1} \in X_{1}$ and $y_{2}$ is the new vertex created in the definition of $G_{1}$. As $\left(G_{1}, p_{1}\right)$ is balanced, no edge-cut in $G_{1} \backslash e_{1}$ separating $x_{1}$ and $y_{2}$ has weight less than $p\left(e_{1}\right)$. Thus, by an undirected version of the Max-Flow Min-Cut theorem [For] there is an integer-valued $\left(x_{1}, y_{2}\right)$-flow $f$ of value $p\left(e_{1}\right)$ in some acyclic orientation $D$ of $G_{1} \backslash e_{1}$ such that $0 \leq f(e) \leq p_{1}(e), e \in E\left(G_{1} \backslash e_{1}\right)$. It is well known that $f$ can be "decomposed" into a collection $\mathbf{P}$ of $p\left(e_{1}\right)$ directed $\left(x_{1}, y_{2}\right)$ paths in $D$. That is, $f=\chi^{\mathbf{P}} \leq p_{1}$. As $B$ is tight, $\chi^{\mathbf{P}}(e)=p(e)$ for every $e \in B \backslash e_{1}$.

Consider the new weight vector $q$ on $E(G)$ defined by:

$$
q(e)= \begin{cases}\chi^{\mathbf{P}}(e), & \text { if } e \in E\left(G\left[X_{1}\right]\right), \\ p(e), & \text { otherwise }\end{cases}
$$


We claim that $(G, q)$ is admissible. That $q$ is eulerian follows from the fact that $\left(G_{2}, p_{2}\right)$ is eulerian and that each path in $\mathbf{P}$ has exactly two odd-degree vertices. To show $q$ is balanced, we first argue as in the previous paragraph, deducing that there exists a collection $\mathbf{Q}$ of exactly $q\left(e_{1}\right)=p\left(e_{1}\right)$ circuits in $G$, each containing $e_{1}$, such that $\chi^{\mathbf{Q}} \leq q$ and $\chi^{\mathbf{Q}}(e)=q(e)$ for every $e \in E\left(G_{1}\right)$ (in fact, we can arrange for each circuit in $\mathbf{Q}$ to be an extension of a corresponding path in P). Let $B^{\prime}$ be an edge-cut in $G$ and let $e^{\prime} \in$ $B^{\prime}$. If $e^{\prime} \in E\left(G_{1}\right)=E\left(G\left[X_{1}\right]\right) \cup B$, then each of the $q\left(e^{\prime}\right)$ circuits in $\mathbf{Q}$ which contain $e^{\prime}$ contains at least one edge in $B^{\prime} \backslash e^{\prime}$. Thus $q\left(B^{\prime} \backslash e^{\prime}\right) \geq q\left(e^{\prime}\right)$. We assume that $e^{\prime} \in E\left(G\left[X_{2}\right]\right)$. Suppose $B^{\prime}$ contains the leader $e_{1}$ of the tight cut $B$. Then $B^{\prime} \triangle B$ is an edge cut with $e^{\prime} \in B^{\prime} \triangle B, e_{1} \notin B^{\prime} \triangle B$, and $q\left(B^{\prime} \backslash e^{\prime}\right) \geq q\left(\left(B^{\prime} \triangle B\right) \backslash e^{\prime}\right)$. So to show that $q\left(B^{\prime} \backslash e^{\prime}\right) \geq q\left(e^{\prime}\right)$ it suffices to show that $q\left(\left(B^{\prime} \triangle B\right) \backslash e^{\prime}\right) \geq q\left(e^{\prime}\right)$. Hence we can assume $e_{1} \notin B^{\prime}$. Let $B^{\prime}=\delta\left(X^{\prime}\right)$ where the set of vertices $X^{\prime}$ is chosen to contain neither endvertex of $e_{1}$. Consider the edge-cut $B^{\prime \prime}=\delta\left(X^{\prime} \cap X_{2}\right)$. Note that $e^{\prime} \in B^{\prime} \cap B^{\prime \prime}$. Let $e \in B^{\prime \prime} \backslash B^{\prime}$. Since $B^{\prime \prime} \backslash B^{\prime}$ consists entirely of followers in the tight cut $B$, each of the $q(e)=p(e)$ circuits $C \in \mathbf{Q}$ which contain $e$ contains no other edge in $B^{\prime \prime} \backslash B^{\prime}$. Since $B^{\prime} \triangle B^{\prime \prime}=\left(B^{\prime \prime} \backslash B^{\prime}\right) \cup\left(B^{\prime} \backslash B^{\prime \prime}\right)$ is an edge-cut and $\left|C \cap\left(B^{\prime \prime} \backslash B^{\prime}\right)\right|=1$, $C$ contains at least one edge in $B^{\prime} \backslash B^{\prime \prime}$. This implies $q\left(B^{\prime} \backslash B^{\prime \prime}\right) \geq q\left(B^{\prime \prime} \backslash B^{\prime}\right)$, whence $q\left(B^{\prime} \backslash e^{\prime}\right) \geq q\left(B^{\prime \prime} \backslash e^{\prime}\right)$. Since $p$ is balanced and coincides with $q$ on $B^{\prime \prime}, q\left(B^{\prime \prime} \backslash e^{\prime}\right)=p\left(B^{\prime \prime} \backslash e^{\prime}\right) \geq p\left(e^{\prime}\right)=q\left(e^{\prime}\right)$. The last two inequalities establish that $q$ is balanced and hence that $q$ is admissible as claimed.

Were $(G, q)$ to have a circuit cover then so would the contra-weighted graph $\left(G_{2}, p_{2}\right)$ (by Proposition 4), a contradiction. Thus $(G, q)$ is contra-weighted. Since $q \leq p$ and $p$ is minimal, we must have $q=p$.

As $D$ is acyclic and $x_{1}$ is a source and $y_{2}$ is a sink, there is an ordering $\left(x_{1}, x_{2}, \ldots, x_{k+1}=y_{2}\right)$ of the vertices in $V\left(G_{1}\right)$ such that all directed arcs $\left(x_{i}, x_{j}\right)$ in $D$ have $i<j$. Thus, $X_{1}=\left\{x_{1}, x_{2}, \ldots, x_{k}\right\}$ and, since $q$ agrees with $\chi^{\mathbf{P}}$ on $E\left(G\left[X_{1}\right]\right)$, all edge-cuts of the form $\delta\left(\left\{x_{1}, x_{2}, \ldots, x_{i}\right\}\right), i=$ $1,2, \ldots, k$, are tight, with $e_{1}$ as their common leader.

\section{Proof of Lemma 1}

As mentioned above, part of this proof is essentially the same as a large part of Seymour's proof of Corollary 1. Unfortunately, Seymour's proof cannot be directly modified into a proof of Lemma 1. The main obstacle is that Seymour relies on a reduction method (vertex "splitting") which, although preserving planarity, can inadvertently introduce $P_{10}$-minors. We use instead a more involved "circuit cover-splicing" argument. An edge-cut $[X, Y]$ is trivial if $|X|=1$ or $|Y|=1$, and is nontrivial otherwise.

Let $(G, p)$ be a minimal positive contra-weighted graph. Our aim is to show that $p$ is $\{1,2\}$-valued.

(4.1) Suppose that $(G, p)$ has a nontrivial tight cut. By Proposition 5 there exist two tight cuts $\delta\left(\left\{x_{1}, x_{2}\right\}\right), \delta\left(\left\{x_{1}\right\}\right)$ with a common tight cut leader $e_{1}=$ $x_{1} y_{1}$. Let $S$ be the set of edges joining $x_{1}$ to $x_{2}$ and let $T=\delta\left(\left\{x_{2}\right\}\right) \backslash S$. Since $\delta\left(\left\{x_{1}\right\}\right)$ and $\delta\left(\left\{x_{1}, x_{2}\right\}\right)$ are both tight, we have $p(S)=p(T)$. Let $\left(G^{\prime}, p^{\prime}\right)$ be obtained from $(G, p)$ by contracting $S$. As $(G, p)$ is admissible, so is $\left(G^{\prime}, p^{\prime}\right)$. Furthermore, $\left(G^{\prime}, p^{\prime}\right)$ has no circuit cover since, by the fact that $p(S)=p(T)$, such a circuit cover is easily modified to be one of $(G, p)$. 
By induction on $|E(G)|$, we can assume there exists a $\{0,1,2\}$-valued weight vector $q^{\prime} \leq p^{\prime}$ such that $\left(G^{\prime}, q^{\prime}\right)$ is contra-weighted. We now extend $q^{\prime}$ into a $\{0,1,2\}$-valued weight vector $q$ for $G$ by defining $q(e)=q^{\prime}(e)$ for $e \in$ $E(G) \backslash S$ and by specifying $q(e)$ for $e \in S$ as follows. If $q(T)$ is odd, then we define $q\left(e_{2}\right)=1$ for some $e_{2} \in S$ and $q(e)=0$ for $e \in S \backslash e_{2}$. If $q(T)$ is even and $|S| \geq 2$, then we define $q\left(e_{2}\right)=q\left(e_{3}\right)=1$ for some $e_{2}, e_{3} \in S$ and $q(e)=0$ for $e \in S \backslash\left\{e_{2}, e_{3}\right\}$. Finally, if $q(T)$ is even and $S=\left\{e_{2}\right\}$, then we define either $q\left(e_{2}\right)=0$ or $q\left(e_{2}\right)=2$ depending on whether or not there exists an edge cut $B$ in $G$ such that $e_{2} \in B$ and $q^{\prime}\left(B \backslash e_{2}\right)=0$. In each case, we have $q(S) \equiv q(T)(\bmod 2)$ ensuring that $(G, q)$ is eulerian. Since $q$ is $\{0,1,2\}$-valued and eulerian, $(G, q)$ is balanced provided that no edge cut $B$ in $G$ contains an edge $e$ with $q(e)=2$ and $q(B \backslash e)=0$ (see Proposition 6). That no such edge cut exists follows from the definition of $q$ on $S$ and the fact that $\left(G^{\prime}, q^{\prime}\right)$ is balanced. Thus $(G, q)$ is admissible. Since $p$ is positive and eulerian, and since $q^{\prime} \leq p^{\prime}$, one easily checks that $q \leq p$. Furthermore, $(G, q)$ has no circuit cover otherwise contracting $S$ would yield a circuit cover of $\left(G^{\prime}, q^{\prime}\right)$. Thus $(G, q)$ is contra-weighted and, by minimality of $p$, we have $q=p$. In this case there is nothing to prove. Hence, we can assume that every tight cut is trivial.

(4.2) Similarly, by contracting one edge of any 2-edge-cut (such a cut must be tight) and using the induction hypothesis, we can assume that $G$ is 3-edgeconnected.

Any edge which is not a follower in any tight cut of $(G, p)$ is called a nonfollower. Let $e$ be an edge in $E(G)$ of maximum weight. We may assume $p(e) \geq 2$ since otherwise $p=1$ (recall that $p \geq 1$ ) and $G$ is eulerian, whence it has a circuit decomposition. By (4.2) and the fact that $p$ is positive, $e$ is a nonfollower.

Let $e_{0}=x y$ be any nonfollower of weight at least 2 such that $p\left(e_{0}\right)$ is as small as possible. Let $r=p\left(e_{0}\right)$. By (4.1), any edge which is a tight cut follower is adjacent to a tight cut leader. This leader must itself be a nonfollower since otherwise, as in the proof of Proposition 2, the symmetric difference of the two tight cuts would be a nontrivial tight cut, contradicting (4.1). Thus any edge of weight at least 2 is either a nonfollower, or is adjacent to a nonfollower (of greater weight). By choice of $e_{0}$ we have the following.

(4.3) Every edge of weight at least 2 either has weight at least $r$ or is a follower in a trivial tight cut whose leader has weight at least $r$.

Define a new weight vector $p^{\prime}$ by $p^{\prime}=p-2 \chi^{e_{0}}$. We claim that $\left(G, p^{\prime}\right)$ is admissible. Since $p\left(e_{0}\right) \geq 2, p^{\prime}$ is nonnegative. As $p$ is eulerian, so is $p^{\prime}$. We now show that $p^{\prime}$ is balanced. Let $B$ be an edge-cut and let $e \in B$. Since $p^{\prime} \leq p$ and $p$ is balanced, then $p^{\prime}(e) \leq p(e) \leq p(B \backslash e)$. We can assume $e_{0} \in B \backslash e$ since otherwise $p(B \backslash e)=p^{\prime}(B \backslash e)$ and we are done. As $e_{0}$ is a nonfollower, we have $p(B \backslash e)-p(e)>0$. Since $p(B)$ is even, this implies $p(B \backslash e)-p(e) \geq 2$. Hence $p^{\prime}(e)=p(e) \leq p(B \backslash e)-2=p^{\prime}(B \backslash e)$. Thus $\left(G, p^{\prime}\right)$ is balanced and hence admissible as claimed.

By minimality of $p$, there exists a circuit cover $\mathbf{L}$ of $\left(G, p^{\prime}\right)$. We write $\mathbf{L}=\mathbf{L}_{1} \cup \mathbf{L}_{2}$ where the circuits in $\mathbf{L}_{1}$ do not contain $e_{0}=x y$ and those in $\mathbf{L}_{2}$ do. Each of the $r-2$ circuits in $L_{2}$ is endowed with an orientation such that $e_{0}$ is traversed from $y$ to $x$.

The next paragraph closely follows Seymour's argument in [Sey1], starting 
with the fifth paragraph of p. 349. For completeness, we reiterate the main points, omitting some details.

We define an auxiliary directed graph $G_{\mathrm{L}}$ with $V\left(G_{\mathrm{L}}\right)=V(G)$. For each $C \in \mathrm{L}_{1}$ and each pair $u, v \in V(C)$ we have an arc $u \rightarrow v$ (this arc is labelled with " $C$ "). For each $C \in \mathrm{L}_{2}$ and each pair $u, v \in V(C)$ which are distinct from $x, y$, we have an arc $u \rightarrow v$ (labelled with " $C$ ") provided that $C$ passes through $y, x, v, u$ in that order (this arc goes the "wrong way" with respect to the orientation of $C)$. As in [Sey1], the fact that $(G, p)$ is balanced implies that there is a directed path from $x$ to $y$ in $G_{\mathrm{L}}$. Let $x=v_{0} \rightarrow v_{1} \rightarrow \cdots \rightarrow v_{k}=y$ be a shortest such path, and let $\left(C_{1}, C_{2}, \ldots, C_{k}\right)$ be the sequence of arc labels along this path. Let $\mathbf{L}^{\prime} \subseteq \mathbf{L}$ denote the underlying set of circuits $\left\{C_{1}, \ldots, C_{k}\right\}$ which appear in the sequence $\left(C_{1}, C_{2}, \ldots, C_{k}\right)$ (repetitions eliminated), and consider the weight vector $\chi^{\mathrm{L}^{\prime}}+2 \chi^{e_{0}}$. Using Proposition 3 and the definition of $G_{\mathbf{L}}$, one can check that $\left(G, \chi^{\mathbf{L}^{\prime}}+2 \chi^{e_{0}}\right)$ is admissible. Suppose that $\mathbf{L}^{\prime}$ is a proper subset of $\mathbf{L}$ so that $\chi^{\mathbf{L}^{\prime}}+2 \chi^{e_{0}}<\chi^{\mathbf{L}}+2 \chi^{e_{0}}=p$. Then $\left(G, \chi^{\mathbf{L}^{\prime}}+2 \chi^{e_{0}}\right)$ has a circuit cover by minimality of $p$. Adjoining the circuits in $\mathbf{L} \backslash \mathbf{L}^{\prime}$ to this circuit cover yields a circuit cover of $\left(G, \chi^{\mathrm{L}}+2 \chi^{e_{0}}\right)=(G, p)$, a contradiction. We conclude that $\mathbf{L}=\mathbf{L}^{\prime}=\left\{C_{1}, C_{2}, \ldots, C_{k}\right\}$.

We now focus on the sequence $\left(v_{0}, C_{1}, v_{1}, C_{2}, \ldots, C_{k}, v_{k}\right)$ of vertices and circuits to determine some structural characteristics of $(G, p)$ and the circuit cover $\mathbf{L}=\mathbf{L}_{1} \cup \mathbf{L}_{2}$ of $\left(G, p-2 \chi^{e_{0}}\right)$.

Let $e \in E(G)$. It follows from the minimality of the length of $x=v_{0} \rightarrow$ $v_{1} \rightarrow \cdots \rightarrow v_{k}=y$ that there are at most two circuits in $\mathrm{L}_{1}$ passing through $e$. Since $\left|\mathbf{L}_{2}\right|=r-2$ we have $p(e) \leq 2+(r-2)=r$. This fact, along with (4.3), implies that every edge in $(G, p)$ of weight at least 2 either has weight exactly $r$ or is adjacent to an edge of weight exactly $r$. This implies the following:

(4.4) Each edge of weight at least 2 has an endvertex $w$ such that either $w=x$, or $w=y$, or $w$ is contained in each of the $r-2$ circuits in $\mathbf{L}_{2}$, as well as two adjacent circuits $C_{i-1}, C_{i} \in \mathrm{L}_{1}$.

Consider the sequence $\left(C_{1}, C_{2}, \ldots, C_{k}\right)$ of circuits which label the arcs of the above $(x, y)$-path in $G_{\mathbf{L}}$. Each circuit in $\mathbf{L}_{2}$ may occur more than once in this sequence. For each $D \in \mathrm{L}_{2}$ we define the nonempty set of indices $I(D):=\left\{i: D=C_{i}\right\}$.

Recall that each $D \in \mathbf{L}_{2}$ is endowed with an orientation. For each $i \in$ $I(D), D$ meets the two vertices and $v_{i}$ and $v_{i-1}$ in that order (starting from $x$ ). (Note that $v_{i} \in V(D)$ does not imply $i \in I(D)$; in particular, $\{0, k\} \cap$ $I(D)=\varnothing$.) The set of vertices $\{x, y\} \cup\left\{v_{i-1}, v_{i}: i \in I(D)\right\}$ partitions $D$ into $2|I(D)|+2$ subpaths which are called the segments of $D$. These segments inherit a natural orientation from $D$. A segment of $D$ starting at $v_{i}$ and ending at $v_{j}$ is denoted by $D\left[v_{i}, v_{j}\right]$. Segments of the form $D\left[v_{i}, v_{i-1}\right]$ where $i \in I(D)$ are called reverse segments of $D$; segments of the form $D\left[v_{i}, v_{j}\right]$ where $0 \leq i<j \leq k$ are called forward segments of $D$; the remaining segment, $D\left[v_{k}, v_{0}\right]=e_{0}$ is called the root segment of $D$.

Let $C_{s}, C_{t} \in \mathrm{L}_{1}$. By the definition of $G_{\mathrm{L}}$ (any two vertices $u, v \in V(C)$, $C \in \mathrm{L}_{1}$, are joined by the two arcs $u \rightarrow v, v \rightarrow u$ ), and by the choice of $v_{0} \rightarrow v_{1} \rightarrow \cdots \rightarrow v_{k}, C_{s}$ and $C_{t}$ are distinct circuits if $s \neq t$, and are vertexdisjoint if $|t-s|>1$. Thus $\chi^{L_{1}}$ is $\{0,1,2\}$-valued and the circuits in $\mathbf{L}_{1}$ form connected "chains" of circuits in $G$. More precisely, a chain is a maximal 
nonempty consecutive sequence $\mathbf{Q}=\left(C_{i+1}, C_{i+2}, \ldots, C_{j}\right)$ of circuits in $\mathbf{L}_{1}$. The vertices $v_{i}$ and $v_{j}$ are called the initial and terminal vertices of $\mathbf{Q}$, and $\mathbf{Q}$ is called a $\left(v_{i}, v_{j}\right)$-chain. Let $\cup \mathbf{Q}$ denote the 2-edge-connected subgraph of $G$ which is the union of the circuits in $\mathbf{Q}$. For each $\left(v_{i}, v_{j}\right)$-chain $\mathbf{Q}$ we arbitrarily choose two $\left(v_{i}, v_{j}\right)$-paths, say $S_{1}$ and $S_{2}$, in $\cup \mathbf{Q}$ such that $\chi^{\left\{S_{1}, S_{2}\right\}} \leq \chi^{\mathbf{Q}}$. The paths $S_{1}, S_{2}$ are called chain segments associated with $\mathbf{Q}$.

We have much flexibility in the choice of chain segments for $\mathbf{Q}$. In fact, for any $\left(v_{i}, v_{j}\right)$-path $S$ in $\cup \mathbf{Q}$, there exists a pair $\left\{S_{1}, S_{2}\right\}$ of chain segments for $\mathbf{Q}$ with $S_{1}=S$. (This follows from the Max-flow Min-cut Theorem and the fact that any $\left\{v_{i}, v_{j}\right\}$-separating cut $B$ has even weight in $\left(\cup \mathbf{Q}, \chi^{\mathbf{Q}}\right)$ whereas $B$ has strictly smaller odd weight in $\left(\cup \mathbf{Q}, \chi^{S}\right)$.) Since the block-graph of $\cup \mathbf{Q}$ is a path, we have the following:

(4.5) For any edge $e \in \bigcup \mathbf{Q}$, there exists a pair $\left\{S_{1}, S_{2}\right\}$ of chain segments for $\mathbf{Q}$ such that $e \in S_{1}$.

Any vertex $v_{s}$, where $i<s<j$, is called an internal vertex of the $\left(v_{i}, v_{j}\right)$ chain $\mathbf{Q}$. Thus, a chain of one circuit has no internal vertices. Any vertex $v_{s}$, $1 \leq s \leq k$, which is not an internal vertex of some chain is called an external vertex of $G$. Thus every external vertex $v_{s}$ either is an initial or terminal vertex of some chain, or each of $C_{s}, C_{s+1}$ belongs to $\mathbf{L}_{2}$. The set of external vertices is exactly the set of initial and terminal vertices of all forward segments, reverse segments and chain segments (collectively called segments).

We define an auxiliary directed graph $H$. The vertices of $H$ are the set of external vertices in $G$. There are three types of arcs in $E(H)$, corresponding to the three types of segments.

(i) For each $\left(v_{i}, f_{j}\right)$-chain $\mathbf{Q}$ in $G$ we have exactly two parallel arcs in $H$ from $v_{i}$ to $v_{j}$. These two arcs correspond to the two chain segments associated with $\mathbf{Q}$.

(ii) For each circuit $D$ in $L_{2}$ and each forward segment $D\left[v_{i}, v_{j}\right]$, we have a corresponding arc $\left(v_{i}, v_{j}\right)$ in $H$.

(iii) For each circuit $D$ in $L_{2}$ and each reverse segment $D\left[v_{i}, v_{i-1}\right]$, we have a corresponding arc $\left(v_{i-1}, v_{i}\right)$ in $H$.

We note that all arcs $\left(v_{i}, v_{j}\right)$ in $E(H)$ have $i<j$ and that there is no arc in $H$ joining $y=v_{k}$ to $x=v_{0}$ (we ignore the root segment). Figure 2 depicts a typical example of a circuit cover $L$ of $\left(G, p^{\prime}\right)$ and the associated directed graph $H$.

For $s=1,2, \ldots, k$, let $K(s)$ denote the set of those $\operatorname{arcs}\left(v_{i}, v_{j}\right)$ with $i<s \leq j$ (this definition makes sense even if $v_{s}$ is not a vertex of $H$ ). We claim that each $K(s)$ is an arc-cut in $H$ of cardinality $r$. As all arcs $\left(v_{i}, v_{j}\right)$ in $H$ have $i<j, K(s)$ is indeed an arc-cut in $H$. Each of the $r-2$ circuits $D$ in $\mathrm{L}_{2}$ contributes exactly one arc (having type (ii)) to $K(s)$, unless $D=C_{s}$, in which case $D$ contributes exactly three arcs to $K(s)$ (one arc of type (iii) and two arcs of type (ii)). Thus $|K(s)|=r$ if $C_{s} \in \mathbf{L}_{2}$. If $C_{s} \in \mathbf{L}_{1}$ then $K(s)$ contains two arcs of type (i) (corresponding to the chain containing $C_{s}$ ) in addition to the $r-2$ arcs of type (ii) contributed by $L_{2}$. Thus $|K(s)|=r$ if $C_{s} \in \mathrm{L}_{1}$, proving our claim.

It follows from the Max-flow Min-cut theorem [For] that the arcs of $H$ can be partitioned into a set of $r$ arc-disjoint directed $(x, y)$-paths $\mathbf{P}=\left\{P_{1}, \ldots, P_{r}\right\}$. We also have the following: 

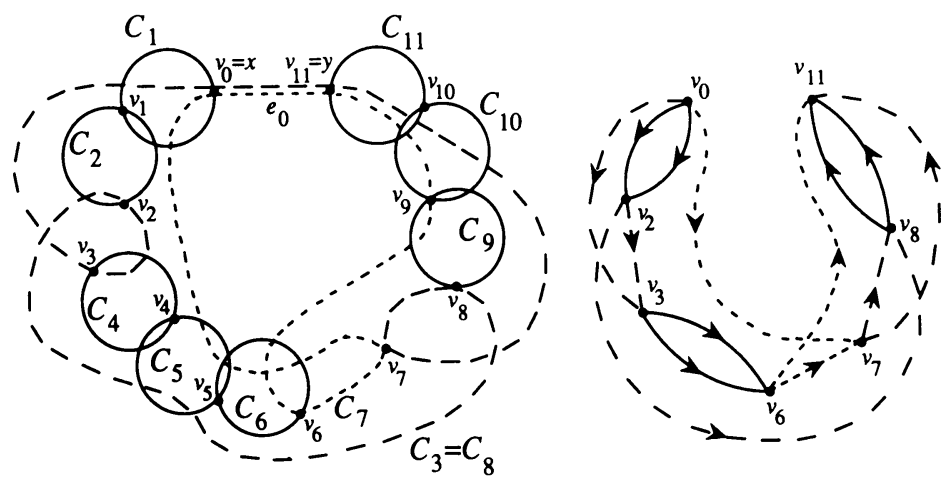

FIGURE 2

(4.6) Each $P_{i} \in \mathbf{P}$ intersects each cut $K(s)$ in exactly one arc.

Each $P_{i} \in \mathbf{P}$ naturally corresponds to an (undirected) $(x, y)$-walk in $G \backslash e_{0}$; traversing an arc in $P_{i}$ corresponds to traversing the corresponding segment in $G$. (Note that the reverse segments are traversed in the "wrong" direction.) Adding the root segment $(y, x)$ to this walk gives a closed walk in $G$ denoted by $W_{i}$. Let $\mathbf{W}=\left\{W_{1}, W_{2}, \ldots, W_{r}\right\}$. We claim the following:

(4.7) No edge is traversed twice along $W_{i}$. Thus each $W_{i}$ is a cycle.

(Recall that a cycle is any edge-disjoint union of circuits.) To prove (4.7), suppose that some edge $e \in E(G)$ is contained in two of the segments, say $S_{1}$ and $S_{2}$, constituting two subwalks in $W_{i}$. Let $s_{1}$ and $s_{2}$ denote the arcs in $H$ corresponding to $S_{1}$ and $S_{2}$. As $S_{1}$ and $S_{2}$ each contain $e, p(e) \geq 2$. Neither $x$ nor $y$ can be an endvertex of $e$ for this would imply that either $K(1)$ or $K(k)$ contains both $s_{1}$ and $s_{2}$, contradicting (4.6). Thus by (4.4), some endvertex $v$ of $e$ is contained in two adjacent circuits $C_{s-1}, C_{s} \in \mathbf{L}_{1}$. These two circuits belong to some chain $\mathbf{Q}$. Each $S_{j}, j=1,2$, is either

(i) a chain segment associated with $\mathbf{Q}$, or

(ii) a segment of the form $D\left[v_{m}, v_{n}\right]$ for some $D \in \mathbf{L}_{2}$.

In case (ii), $v \neq v_{m}, v_{n}$ since $v$ is not an external vertex. Thus in $G_{\mathbf{L}}$ we have $v_{n} \rightarrow v \rightarrow v_{m}$. By the minimality of the sequence $v_{0} \rightarrow v_{1} \rightarrow \cdots \rightarrow v_{k}$, this implies $m<s<n$. In either case, $s_{j}$ belongs to $K(s), j=1,2$, contradicting (4.6), and proving (4.7).

The cycles $W_{i} \in \mathbf{W}$ might not be circuits since consecutive segments in $W_{i}$ might have many vertices in common. However, only "nearby" segments can overlap as the following attests.

(4.8) Let $\left(v_{a}, v_{b}\right)$ and $\left(v_{c}, v_{d}\right)$ be two arcs in $H$ such that $a<b<c<d$. Then the two corresponding segments $S_{a, b}, S_{c, d} \subseteq G$ are vertex-disjoint.

Let $v \in V\left(S_{a, b}\right)$. If $S_{a, b}$ is not a chain segment, then since $b<k, S_{a, b}$ is either the forward segment $C_{b}\left[v_{a}, v_{b}\right]$ or the reverse segment $C_{b}\left[v_{b}, v_{b-1}\right]$. In each case, either $v=v_{b-1}$ or $G_{\mathbf{L}}$ contains the arc $v_{b-1} \rightarrow v$. If $S_{a, b}$ is a chain segment, then $v$ lies on some circuit $C_{\alpha} \in \mathrm{L}_{1}$ where $a+1 \leq \alpha \leq b$. Here, either $v=v_{\alpha-1}$ or the arc $v_{\alpha-1} \rightarrow v$ is contained in $G_{\mathrm{L}}$. In any case, there exists an $s<b$, such that either $v=v_{s}$ or the arc $v_{s} \rightarrow v$ is contained in $G_{\mathrm{L}}$. Similarly, if $u \in V\left(S_{c, d}\right)$, then there exists a $t>c$ such that either $u=v_{t}$ or the arc $u \rightarrow v_{t}$ is contained in $G_{\mathrm{L}}$. If $v=u$, then in $G_{\mathrm{L}}$ we have either 
$v_{s}=v=u \rightarrow v_{t}$ or $v_{s} \rightarrow v=u=v_{t}$ or $v_{s} \rightarrow v=u \rightarrow v_{t}$, where $s \leq t-3$, contradicting the minimality of the sequence $v_{0} \rightarrow v_{1} \rightarrow \cdots \rightarrow v_{k}$. Thus $S_{a, b}$ and $S_{c, d}$ are vertex-disjoint, proving (4.8).

By construction of $\mathbf{W}, \chi^{\mathbf{W}}\left(e_{0}\right)=r$. Furthermore, $\chi^{\mathbf{W}}(e) \leq \chi^{\mathbf{L}}(e)=p(e)$ for every edge $e$ belonging to some circuit in $\mathbf{L}_{1}$. Since $\mathbf{W}$ constitutes a partition of all forward, reverse, root, and chain segments, the following is true.

(4.9) We have $\chi^{\mathrm{W}} \leq p$, with equality on all edges not belonging to some circuit in $L_{1}$.

We now foreshadow the completion of this proof. For each chain $\mathbf{Q}$ in $G$, we shall define an admissible $\{0,1,2\}$-valued vector $q_{\mathbf{Q}}$ such that $\chi^{\mathbf{Q}} \leq q_{\mathbf{Q}} \leq$ $p$. If $\left(G, q_{\mathbf{Q}}\right)$ has a circuit cover for each chain $\mathbf{Q}$, then we can obtain a circuit cover of $(G, p)$ by "splicing" these circuit covers together (using $\mathbf{W}$ ), a contradiction. Thus $\left(G, q_{\mathbf{Q}}\right)$ is contra-weighted for some chain $\mathbf{Q}$, implying, by minimality of $p$, that $p=q_{\mathbf{Q}}$, whence we shall have proven Lemma 1 .

Let $\mathbf{Q}$ be a $\left(v_{i}, v_{j}\right)$-chain and let $S_{1}, S_{2}$ be the two chain segments associated with $\mathbf{Q}$. Exactly two cycles in $\mathbf{W}$, say $W_{1}$ and $W_{2}$, contain the chain segments $S_{1}$ and $S_{2}$, respectively. We define a weight vector $q_{\mathrm{Q}}$ on $E(G)$ as follows:

$$
q_{\mathbf{Q}}=\chi^{\mathbf{Q}}+\chi^{\left\{W_{1} \backslash S_{1}, W_{2} \backslash S_{2}\right\}} .
$$

The path $W_{s} \backslash S_{s}$ is edge-disjoint from the chain segment $S_{s}$, for $s=1,2$, by (4.7). This statement holds true regardless of which particular pair $\left\{S_{1}, S_{2}\right\}$ of chain segments were initially chosen for $Q$ (just prior to (4.5)). Because of the flexibility in our choice of $\left\{S_{1}, S_{2}\right\}$ described in (4.5) and because of (4.8), we may conclude that the entire subgraph $\cup Q$ is edge-disjoint from $W_{s} \backslash S_{s}$, $s=1,2$. By the definition of $\mathbf{Q}$ and the facts $\mathbf{L}_{1} \subseteq \mathbf{L}$ and $p^{\prime} \leq p$ and by (4.9) we have that $\chi^{\mathbf{Q}} \leq p, \chi^{\mathbf{W}} \leq p$ and both $\chi^{\mathbf{Q}}$ and $\chi^{\left\{W_{1}, W_{2}\right\}}$ are $\{0,1,2\}$ valued. Hence $q_{\mathbf{Q}} \leq p$ and is $\{0,1,2\}$-valued. Note that $q_{\mathbf{Q}}\left(e_{0}\right)=2$. Since $W_{1}$ and $W_{2}$ are cycles, $v_{i}$ and $v_{j}$ are the only vertices of odd degree in each of the subgraphs $W_{1} \backslash S_{1}$ and $W_{2} \backslash S_{2}$. It follows that $\chi^{\left\{W_{1} \backslash S_{1}, W_{2} \backslash S_{2}\right\}}$ is eulerian. By Proposition $3, \chi^{\mathbf{Q}}$ is eulerian, so $q_{\mathrm{Q}}$ is eulerian. As $q_{\mathrm{Q}}$ is eulerian, $\{0,1,2\}$ valued and has as support the 2-edge-connected subgraph $\cup \mathbf{Q} \cup W_{1} \cup W_{2}, q_{\mathbf{Q}}$ is admissible by Proposition 6 .

Suppose $\left(G, q_{\mathbf{Q}}\right)$ has a circuit cover $\mathbf{X}_{\mathbf{Q}}$ for each chain $\mathbf{Q}$. It remains to show that we can splice these circuit covers together and obtain a circuit cover $\mathbf{X}$ of $(G, p)$. Roughly, $\mathbf{X}$ shall consist of a modification of the cycles in $\mathbf{W}$ together with a subset $\mathbf{Y}_{\mathbf{Q}}$ of each circuit cover $\mathbf{X}_{\mathbf{Q}}$.

Let $\mathbf{Q}=\left(C_{i+1}, C_{i+2}, \ldots, C_{j}\right)$ be any $\left(v_{i}, v_{j}\right)$-chain and let $W_{1}, W_{2} \in \mathbf{W}$ be as above. For $s=1,2$, let $v_{i}^{s}$ and $v_{j}^{s}$ denote the first and last vertices, respectively, of $\cup \mathrm{Q}$ encountered when $W_{s}$ is traversed (in the usual direction) starting at $x$. The three vertices $v_{i}^{1}, v_{i}^{2}$, and $v_{i}$ might not be distinct (and similarly for $v_{j}^{1}, v_{j}^{2}$, and $v_{j}$ ). For example, we know that $v_{0}^{1}=v_{0}^{2}=v_{0}=x$. The vertices in $\left\{v_{i}^{1}, v_{i}^{2}, v_{j}^{1}, v_{j}^{2}\right\}$ are called the connector vertices of $\mathbf{Q}$. If $i>0$, then the last edge in the subtrail $W_{s}\left[x, v_{i}^{s}\right]$ is denoted by $e_{i}^{s}, s=1,2$. If $i=0$, then we define $e_{i}^{s}=e_{0}, s=1,2$. Similarly, $e_{j}^{s}$ is defined to be either $e_{0}$ (if $j=k$ ) or the first edge in the subtrail $W_{s}\left[v_{j}^{s}, y\right], s=1,2$. The edges in $\left\{e_{i}^{1}, e_{i}^{2}, e_{j}^{1}, e_{j}^{2}\right\}$ are called the connector edges of $\mathbf{Q}$.

Let $i>0$ and let $S_{s}^{-}$denote the segment in $W_{s}\left[x, v_{i}\right]$ which terminates 


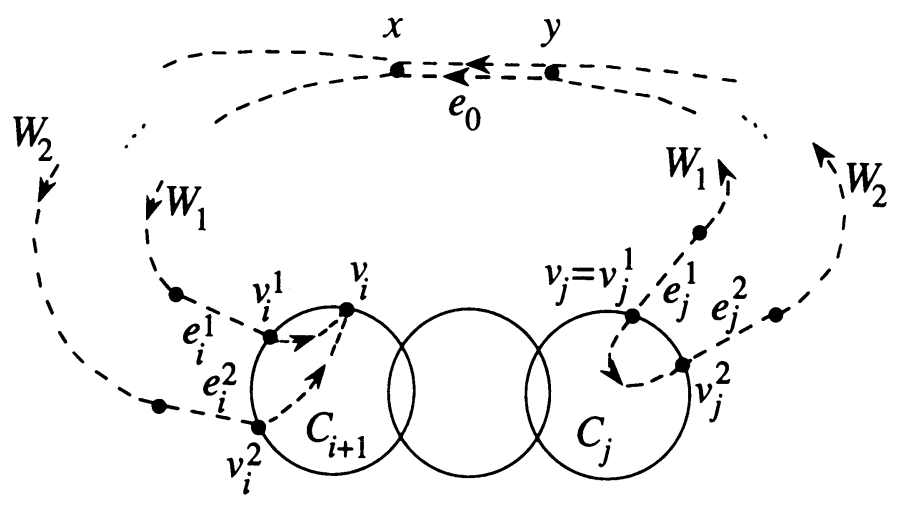

FIGURE 3

at $v_{i}, s=1,2$. Applying (4.8), all segments in $W_{s}\left[x, v_{i}\right] \backslash S_{s}^{-}$are vertexdisjoint from the chain segments $S_{1}$ and $S_{2}$. Because of the arbitrary choice of chain segments for $\mathbf{Q}$, and by (4.5), $W_{s}\left[x, v_{i}\right] \backslash S_{s}^{-}$is vertex-disjoint from all of $\cup \mathbf{Q}, i=1,2$. Also, by (4.8), $W_{1}\left[x, v_{i}\right] \cup W_{2}\left[x, v_{i}\right]$ is vertex-disjoint from $W_{1}\left[v_{j}, y\right] \cup W_{2}\left[v_{j}, y\right]$. Thus, by definition of connector edges, we have $e_{i}^{s} \in S_{s}^{-}, s=1,2$, and that $\left\{e_{i}^{1}, e_{i}^{2}\right\}$ is an edge-cut in $\cup Q \cup W_{1}[x, y] \cup$ $W_{2}[x, y]$ separating the vertices in $W_{1}\left[x, v_{i}^{1}\right] \cup W_{2}\left[x, v_{i}^{2}\right]-\left\{v_{i}^{1}, v_{i}^{2}\right\}$ from those in $\cup Q \cup W_{1}\left[v_{i}^{1}, y\right] \cup W_{2}\left[v_{i}^{2}, y\right]$. Since $S_{1}^{-}$and $S_{2}^{-}$are distinct segments contained in $C_{i} \in \mathrm{L}_{2}$ we have $e_{i}^{1} \neq e_{i}^{2}$. We summarize as follows.

(4.10) If $i>0$, then $\left\{e_{i}^{1}, e_{i}^{2}\right\}$ is a 2-edge-cut in $\left(\cup \mathrm{Q} \cup W_{1} \cup W_{2}\right) \backslash e_{0}$; similarly, if $j<k$, then $\left\{e_{j}^{1}, e_{j}^{2}\right\}$ is a 2-edge-cut in ( $\left.\cup \mathbf{Q} \cup W_{1} \cup W_{2}\right) \backslash e_{0}$ (see Figure 3).

Let $\cup \mathbf{Q}^{+}$denote the connected component of

$$
\left(\bigcup \mathbf{Q} \cup W_{1} \cup W_{2}\right) \backslash\left\{e_{i}^{1}, e_{i}^{2}, e_{j}^{1}, e_{j}^{2}\right\}
$$

which contains the connector vertices. Thus $\cup \mathbf{Q}^{+}$is the union of $\cup \mathbf{Q}$ and the $\left(v_{i}^{1}, v_{i}, v_{i}^{2}\right)$-subpath of $C_{i}$ and the $\left(v_{j}^{1}, v_{j}, v_{j}^{2}\right)$-subpath of $C_{j+1}$ (if $i=0$ or $j=k$, then we use the empty path).

Note that $\mathbf{X}_{\mathbf{Q}}$ is a circuit cover of $\left(\cup \mathbf{Q} \cup W_{1} \cup W_{2}, q_{\mathbf{Q}}\right)$ (see Figure 3). Let $A_{1}, A_{2}$ be the two circuits in $\mathrm{X}_{\mathrm{Q}}$ which contain $e_{0}$. By (4.10), $A_{1}$ contains exactly one edge from each of $\left\{e_{i}^{1}, e_{i}^{2}\right\}$ and $\left\{e_{j}^{1}, e_{j}^{2}\right\} ; A_{2}$ contains the remaining two connector edges. We relabel $A_{1}, A_{2}$ so that $e_{i}^{1} \in E\left(A_{1}\right)$ and $e_{i}^{2} \in E\left(A_{2}\right)$. Every circuit in $\mathrm{X}_{\mathbf{Q}} \backslash\left\{A_{1}, A_{2}\right\}$ is either contained wholly in $\cup \mathbf{Q}^{+}$or is vertexdisjoint from $\cup Q^{+}$. We denote the subset of circuits of the former type by $\mathbf{Y}_{\mathbf{Q}}$.

We recall that a cycle cover of $(G, p)$ is a multiset A of cycles in $G$ such that $\chi^{A}=p$. For example, any circuit cover of $(G, p)$ is also a cycle cover of $(G, p)$. Conversely, by decomposing the cycles in a cycle cover of $(G, p)$, one obtains a circuit cover of $(G, p)$. We aim to produce a cycle cover of $(G, p)$. Let $\mathbf{Y}$ denote the union of $\mathbf{Y}_{\mathbf{Q}}$ over all chains $\mathbf{Q}$. Although $\mathbf{W}$ is a cycle cover of $\left(G, \chi^{\mathbf{W}}\right), \mathbf{Y} \cup \mathbf{W}$ is not quite a cycle cover of $(G, p)$. We must still modify the cycles in $\mathbf{W}$ so that they "mesh" correctly with $\mathbf{Y}_{\mathbf{Q}}$ within each chain $\mathbf{Q}$.

For each chain $\mathbf{Q}, \mathbf{Y}_{\mathbf{Q}}$ is a circuit cover of $\left(G, \chi^{\mathbf{Q}}+\chi^{\left\{W_{1}^{+} \backslash S_{1}, W_{2}^{+} \backslash S_{2}\right\}}-\right.$ $\left.\chi^{\left\{A_{1}^{+}, A_{2}^{+}\right\}}\right)$, where $W_{s}^{+}$and $A_{s}^{+}$denote $W_{s} \cap \cup \mathbf{Q}^{+}$and $A_{s} \cap \cup \mathbf{Q}^{+}$, respectively 


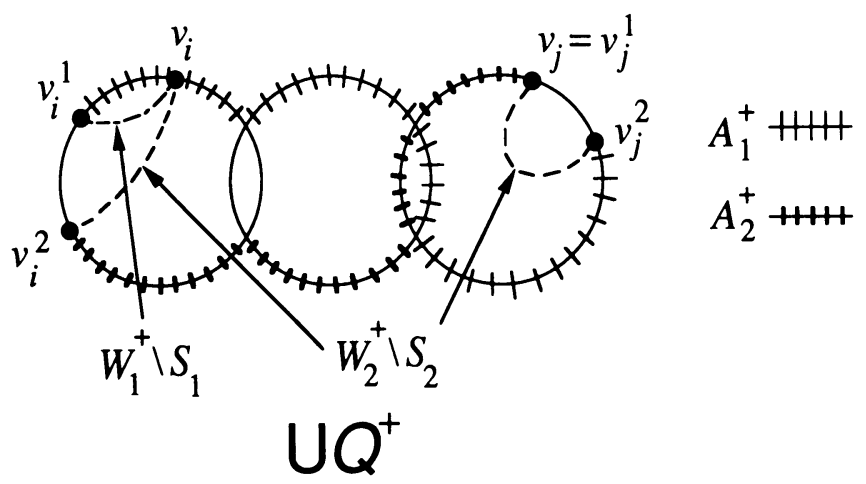

FIGURE 4

(see Figure 4). We modify the two cycles $W_{1}, W_{2} \in \mathbf{W}$ in one of two ways, depending on which of $e_{j}^{1}, e_{j}^{2}$ is an edge of $A_{1}$ (Figure 4 depicts the second possibility). (Recall that $e_{i}^{1} \in E\left(A_{1}\right)$.) If $e_{j}^{1} \in E\left(A_{1}\right)$, then $e_{j}^{2} \in E\left(A_{2}\right)$ and we modify $W_{s}$ by replacing the $\left(v_{i}^{s}, v_{j}^{s}\right)$-subpath $W_{s}^{+}$of $W_{s}$ with the $\left(v_{i}^{s}, v_{j}^{s}\right)$ path $A_{s}^{+}, s=1,2$. If $e_{j}^{2} \in E\left(A_{1}\right)$, then $e_{j}^{1} \in E\left(A_{2}\right)$ and we modify $W_{s}$ by replacing the $\left(v_{i}^{s}, v_{j}^{s}\right)$-subpath $W_{s}^{+}$of $W_{s}$ with the $\left(v_{i}^{s}, v_{j}^{3-s}\right)$-path $A_{s}^{+}$, $s=1,2$, and then interchanging the $\left(v_{j}^{1}, y\right)$-subpath of $W_{1}$ with the $\left(v_{j}^{2}, y\right)$ subpath of $W_{2}$. In either case, each of the resulting two subgraphs are cycles that can take the places of $W_{1}$ and $W_{2}$ in $\mathbf{W}$. After this modification we have

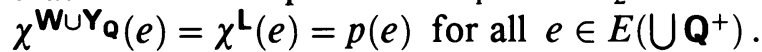

We perform the modification of $\mathbf{W}$ as described in the previous paragraph for every chain $\mathbf{Q}$ in $G$ (in any order). By (4.9) and the observations of the previous paragraph, $\mathbf{W} \cup \mathbf{Y}$ is a cycle cover of $(G, p)$, as required.

\section{Proof of Lemma 2}

We shall need the following lemma which was essentially proved by Ellingham [Ell].

Lemma 3. Let $H$ be a simple cubic graph which has a perfect matching $M$ such that the 2-factor $H \backslash M$ has exactly two components (which are circuits), and every edge in $M$ has one endvertex in each of these circuits. If $H$ does not have a proper 3-edge-coloring, then there exists a subset $S \subseteq M$ such that $H \backslash S$ is a subdivision of Petersen's graph.

When a weight vector $p$ is $\{0,1,2\}$-valued, the admissibility conditions (1.2) degenerate slightly. The set of edges of weight $i$ in $(G, p)$ is denoted $E_{i}$.

Proposition 6. $A$ weight vector $p: E(G) \rightarrow\{0,1,2\}$ is admissible if and only if both of the following hold:

(1) (balance) $G$ has no edge cut containing exactly one positive-weight edge, and

(2) (eulericity) $E_{1}$ is a cycle in $G$.

We note that if (2) holds and (1) fails then the positive-weight edge has weight 2 . 
The following proof of Lemma 2 is a generalization of that given by Alspach and Zhang [Als], which was for cubic graphs only. Since there does not appear to be an analog of Lemma 3 for graphs with higher-degree vertices, it is critical that we reduce to the cubic graph case. The main difficulty here turns out to be the elimination of vertices of degree 4 in minimal contra-weighted graphs.

Let $(G, p)$ be a $\{1,2\}$-valued minimal contra-weighted graph. We aim to show that $(G, p)$ is a blistered Petersen graph. As in the proof of Lemma 1, our first step is to eliminate 2-edge-cuts and nontrivial tight cuts.

(5.1) We can assume $G$ has no vertices of degree 2. If $x$ is such a vertex then we contract one of its incident edges, obtaining $\left(G^{\prime}, p^{\prime}\right)$. By induction on $|E(G)|$, there exists a blistered $P_{10},\left(G^{\prime}, q^{\prime}\right)$, with $q^{\prime} \leq p^{\prime}$. By applying (ii) of (2.1) to $\left(G^{\prime}, p^{\prime}\right)$, we can obtain a blistered $P_{10},(G, q)$, with $q \leq p$, and we are done.

(5.2) We can assume $(G, p)$ has no nontrivial tight cuts. Suppose $G$ has a nontrivial tight cut. There exist two tight cuts $\delta\left(\left\{x_{1}, x_{2}\right\}\right), \delta\left(\left\{x_{1}\right\}\right)$ with a common tight cut leader $e_{1}=x_{1} y_{1}$ by Proposition 5. By (5.1), $x_{1}$ and $x_{2}$ have degree at least 3 . Since $p$ is $\{1,2\}$-valued, it must be the case that $p\left(e_{1}\right)=2$, and that there are two parallel edges of weight $1, e_{2}$ and $e_{3}$, joining $x_{1}$ to $x_{2}$, and that no other edges meet $x_{1}$. We now replace $e_{2}$ and $e_{3}$ with a single edge of weight 2, and argue as in (5.1), applying either (ii) or (iii) of (2.1).

It follows from (5.1), (5.2), and Proposition (6.1) that $G$ is 3-edge-connected. We define $E_{1}$ and $E_{2}$ as above. By Proposition $6, E_{1}$ is a cycle.

The next two paragraphs are specializations of arguments presented in the proof of Lemma 1. We include them for completeness. If $p=\mathbf{1}$, then $(G, p)$ is not contra-weighted, since $G$ is eulerian. Let $e_{0}$ be an arbitrary edge of weight 2 and let $p^{\prime}=p-2 \chi^{e_{0}}$. By Proposition 6 and since $G$ is 3-edge-connected, $\left(G, p^{\prime}\right)$ is admissible. By minimality of $p,\left(G, p^{\prime}\right)$ has a circuit cover.

Let $\mathbf{L}$ be any circuit cover of $\left(G, p^{\prime}\right)$, and let $\mathbf{L}^{\prime}$ be a minimal subset of $\mathbf{L}$ such that $\left(G, \chi^{\mathrm{L}^{\prime}}+2 \chi^{e_{0}}\right)$ is admissible. (By Proposition 6 , this is equivalent to requiring that $U \mathrm{~L}^{\prime}+e_{0}$ be a bridgeless subgraph of $G$.) If $\left(G, \chi^{\mathrm{L}^{\prime}}+2 \chi^{e_{0}}\right)$ were to have a circuit cover, then adjoining $\mathbf{L}-\mathbf{L}^{\prime}$ to this circuit cover would yield a circuit cover of $(G, p)$, a contradiction. Thus $\left(G, \chi^{L^{\prime}}+2 \chi^{e_{0}}\right)$ is a contra-weighted graph. By minimality of $p$, we have $\mathbf{L}=\mathbf{L}^{\prime}$. It follows that $\mathbf{L}=\left\{C_{1}, C_{2}, \ldots, C_{k}\right\}$ where $C_{i}$ and $C_{j}$ intersect (in at least one vertex) if and only if $|i-j| \leq 1$. Furthermore, $C_{i}$ intersects with $e_{0}$ (at a vertex) if and only if $i=1$ or $i=k$. Using terminology from the proof of Lemma 1, we have the following.

(5.3) Every circuit cover of $\left(G, p^{\prime}\right)$ consists of a single $(x, y)$-chain of circuits, where $x$ and $y$ are the endvertices of $e_{0}$ (see Figure 5). A $k$ cycle cover of $(G, p)$ is a multiset of at most $k$ cycles which covers each edge $e \in E$ exactly $p(e)$ times. Let $D_{0}=\bigcup\left\{C_{i}: i\right.$ is even $\}$ and let $D_{1}=\bigcup\left\{C_{i}: i\right.$ is odd $\}$. Each $D_{i}$ is a cycle in $G$ and $\left\{D_{0}, D_{1}\right\}$ is a 2 cycle cover of $\left(G, p^{\prime}\right)$. Recall that $E_{1}=p^{-1}(1)$ and $E_{2}=p^{-1}(2)$. Consider the contracted graph $G / E_{1}$, and let $D_{i} / E_{1}$ denote the cycle in $G / E_{1}$ which is induced by the edge set $D_{i} \cap E_{2}=E_{2} \backslash e_{0}$. Then $\left\{D_{0} / E_{1}, D_{1} / E_{1}\right\}$ is a 2 cycle cover of $\left(G / E_{1}, 2 \chi^{E_{2} \backslash e_{0}}\right)$. Thus, $D_{0} / E_{1}=D_{1} / E_{1}=E\left(G / E_{1} \backslash e_{0}\right)$ so $G / E_{1} \backslash e_{0}$ is eulerian. Since $e_{0}$ is an arbitrary edge in $E_{2}$, there are exactly two possibilities for $G / E_{1}$ :

(5.4) $G / E_{1}$ contains exactly one vertex, and every edge of $G / E_{1}$ is a loop. 


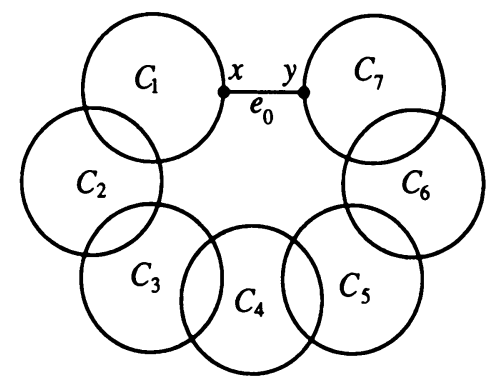

FIGURE 5

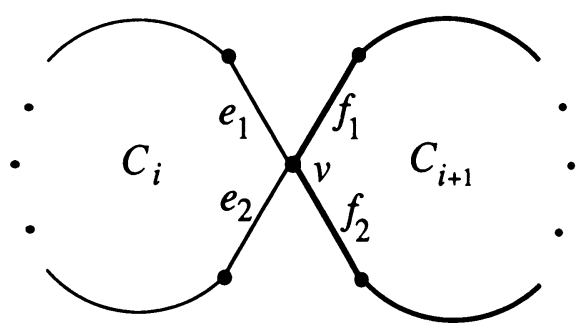

FIGURE 6

(5.5) $G / E_{1}$ contains exactly two vertices, both vertices have odd degree, and every edge of $G / E_{1}$ joins these two vertices.

Suppose that (5.4) is the case. Then there exists an $(x, y)$-path $P$ in $E_{1}$. Let $C$ be the circuit $P+e_{0}$ in $G$. Then $\left\{D_{0} \Delta C, D_{1} \triangle C\right\}$ is a cycle cover of $(G, p)$, a contradiction. Thus $(5.5)$ is the case.

Let $\mathbf{L}$ be a circuit cover of $\left(G, p^{\prime}\right)$. By (5.1) and (5.3), every vertex in $V(G) \backslash\{x, y\}$ is contained in exactly two (consecutive) circuits in $\mathbf{L}$. Thus every vertex in $G$ is either cubic (degree 3 ) or quartic (degree 4). Each cubic vertex is adjacent with exactly one edge in $E_{2}$ and two edges in $E_{1}$. Each quartic vertex is adjacent with exactly four edges in $E_{1}$. Both $x$ and $y$ are cubic vertices. We write $V(G)=V_{3} \cup V_{4}$ where $V_{i}$ denotes the set of vertices of degree $i$ in $G$.

Since $p$ is eulerian, each of the two connected components induced by $E_{1}$ is an eulerian subgraph of $G$ which is either a circuit or a subdivision of some connected 4-regular graph.

We intend to establish that $V_{4}=\varnothing$ and hence that $G$ is a cubic graph. Suppose that $v \in V_{4}$. Let $e_{0} \in E_{2}$ be arbitrary and let $\mathbf{L}$ be a circuit cover of $\left(G, p^{\prime}\right)=\left(G, p-2 \chi^{e_{0}}\right)$ of maximum possible cardinality. By (5.3), $\mathbf{L}$ is an $(x, y)$-chain $\left\{C_{1}, C_{2}, \ldots, C_{k}\right\}$. Thus $v \in V\left(C_{i}\right) \cap V\left(C_{i+1}\right)$ for some unique $i \in\{1,2, \ldots, k-1\}$. Let $\left\{e_{1}, e_{2}\right\}$ be the two edges in $C_{i}$ incident with $v$, and let $\left\{f_{1}, f_{2}\right\}$ be the two edges in $C_{i+1}$ incident with $v$ (see Figure 6).

Consider the subgraph $J:=C_{i} \cup C_{i+1}$ of $G$. There must be some vertex in $V\left(C_{i}\right) \cap V\left(C_{i+1}\right)$ which is different from $v$ for, otherwise, $\left\{e_{0}, e_{1}, e_{2}\right\}$ would be a nontrivial tight cut in $(G, p)$, contradicting (5.2), or else $i=1$ and $C_{1}$ and $C_{2}$ are 2-gons, in which case we obtain a contradiction to the choice of $(G, p)$. Thus $J$ is 2-connected. Suppose that $E\left(C_{i} \cap C_{i+1}\right)=\varnothing$. Then $J$ is eulerian. Since $J$ is 2 -connected, there is a circuit $C$ in $J$ such that $\left(\mathbf{L} \backslash\left\{C_{i}, C_{i+1}\right\}\right) \cup\{C\}$ is an $(x, y)$-chain. The union of this chain with $e_{0}$ is 2- 
connected. Since $(G, p)$ and $\left(G, \chi^{J-C}\right)$ are eulerian, so is $\left(G, p-\chi^{J-C}\right)$. By construction, $p-\chi^{J-C}$ is also balanced. Thus, by Proposition $6,\left(G, p-\chi^{J-C}\right)$ is admissible so, by minimality of $p,\left(G, p-\chi^{J-C}\right)$ has a circuit cover. The union of this circuit cover with the cycle $\{J-C\}$ is a cycle cover of $(G, p)$, a contradiction. Thus $E\left(C_{i}\right) \cap E\left(C_{i+1}\right) \neq \varnothing$.

A subcycle is a subset of a cycle which is also a cycle. Let $r=\chi^{\left\{C_{i}, C_{i+1}\right\}}$, let $F_{1}=r^{-1}(1)=C_{i} \triangle C_{i+1}$ and let $F_{2}=r^{-1}(2)=C_{i} \cap C_{i+1}$. Let $C$ be any subcycle of the cycle $F_{1}$. Like $\left\{C_{i}, C_{i+1}\right\},\left\{C_{i} \triangle C, C_{i+1} \triangle C\right\}$ is a 2 cycle cover of $(G, r)$. Hence $\mathrm{L}_{C}:=\left(\mathbf{L} \backslash\left\{C_{i}, C_{i+1}\right\}\right) \cup\left\{C_{i} \triangle C, C_{i+1} \Delta C\right\}$ is a cycle cover of $\left(G, p^{\prime}\right)$ (in [God2], the transformation $\mathbf{L} \rightarrow \mathbf{L}_{C}$ is called a pivot of $\left\{C_{i}, C_{i+1}\right\}$ on $C$ ). Note that if $C$ is the empty cycle, then $\mathbf{L}_{C}=\mathbf{L}$. Since $F_{2}$ is not empty, $C$ is different from both $C_{i}$ and $C_{i+1}$, so neither $C_{i} \triangle C$ nor $C_{i+1} \Delta C$ is the empty cycle. By maximality of $|\mathrm{L}|$, we have $\left|\mathrm{L}_{C}\right|=|\mathrm{L}|$, so each of the cycles $C_{i} \triangle C$ and $C_{i+1} \triangle C$ is a circuit. Thus $\mathrm{L}_{C}$ is a circuit cover of $\left(G, p^{\prime}\right)$ which, by $(5.3)$, must be an $(x, y)$-chain of circuits.

A block in a graph $H$ is a maximal 2-connected subgraph of $H$. The blocks of $H$ induce a partition of $E(H)$. In the following two paragraphs we compare the block structures of the cycles $F_{1}$ and $E_{1}$. In general these two cycles are different, since any edge in $E\left(C_{i-1} \cap C_{i}\right)$ is in $F_{1} \cap E_{2}$. However, we shall see that all but one of the blocks of $F_{1}$ is also a block of $E_{1}$. Furthermore, we shall see that the quartic vertex $v$ is a cut-vertex of $F_{1}$, and hence of $E_{1}$.

Let $v_{i-1}$ be any vertex in $V\left(C_{i-1}\right) \cap V\left(C_{i}\right)$, and let $v_{i+1}$ be any vertex in $V\left(C_{i+1}\right) \cap V\left(C_{i+2}\right)$ (here, we temporarily define $\left.C_{0}=C_{k+1}=e_{0}\right)$. Then $v_{i-1}, v_{i+1}$ are vertices of degree 2 in $F_{1}$. Let $C$ be any subcycle of $F_{1}$ which contains one of these two vertices, say $v_{i-1}$. Then $C$ must also contain $v_{i+1}$, for otherwise the circuit $C_{i+1} \Delta C$ would contain both $v_{i+1}$ and $v_{i-1}$, contradicting the fact that $\mathbf{L}_{C}$ is an $(x, y)$-chain of circuits. Hence every subcycle of $F_{1}$ contains either all or none of the vertices in $\left(V\left(C_{i-1}\right) \cap V\left(C_{i}\right)\right) \cup\left(V\left(C_{i+1}\right) \cap\right.$ $\left.V\left(C_{i+2}\right)\right)$. This is true, in particular, when the subcycle $C$ of $F_{1}$ is a circuit. Thus all of these vertices belong to a single block $B$ of $F_{1}$. It follows that each block of $F_{1} \backslash B$ is vertex-disjoint from each circuit in $\mathbf{L} \backslash\left\{C_{i}, C_{i+1}\right\}$. Thus we have shown the following.

(5.6) There exists a block $B$ in $F_{1}$ such that every block of $F_{1} \backslash B$ is also a block of $E_{1}$.

Let $C$ be any circuit in $F_{1}$ containing the quartic vertex $v$ (see Figure 6). Then $C$ must contain exactly one edge from $\left\{e_{1}, e_{2}\right\}$ and one edge from $\left\{f_{1}, f_{2}\right\}$, for otherwise $v$ would be a vertex of degree 4 in either $C_{i} \triangle C$ or $C_{i+1} \triangle C$, contradicting the fact that they are circuits. Thus $e_{1}$ and $e_{2}$ belong to distinct blocks of $F_{1}$, and $v$ is a cut-vertex of $F_{1}$. By (5.6) we have the following.

(5.7) The quartic vertex $v$ is a cut-vertex of $E_{1}$. By interchanging the labels of $f_{1}$ and $f_{2}$ if necessary, we may assume that, for $i=1,2, e_{i}$ and $f_{i}$ belong to the same block of $E_{1}$, whereas $e_{1}$ and $e_{2}$ (respectively $f_{1}$ and $f_{2}$ ) belong to distinct blocks of $E_{1}$.

We define a new weighted graph $\left(G^{v}, p^{v}\right)$ from $(G, p)$ by replacing $v$ with two new (cubic) vertices, $v_{1}$ and $v_{2}$, such that, for $i=1,2, v_{i}$ is incident with both $e_{i}$ and $f_{i}$. A new edge $e_{v}$ of weight 2 joins $v_{1}$ and $v_{2}$ (see Figure $7)$. Thus $E\left(G^{v}\right)=E_{1} \cup E_{2} \cup\left\{e_{v}\right\}$. 


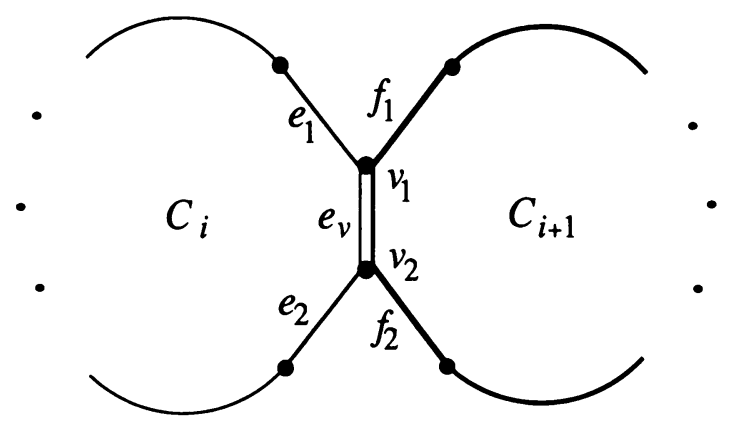

FIGURE 7

The definition of $\left(G^{v}, p^{v}\right)$ depends only on the block structure of $E_{1}$ and the quartic vertex $v$, and is independent of the choice of $\mathbf{L}$ and, indeed, the choice of $e_{0}$. By (5.5) and (5.7), $E_{1}$ induces exactly three connected components in $G^{v}$. A minor modification of $\mathbf{L}$, as depicted in Figure 7, yields a 2-cycle cover $\left\{D_{0}^{v}, D_{1}^{v}\right\}$ of $\left(G^{v}, p^{v}-2 \chi^{e_{0}}\right)$. As in the derivation of $(5.5),\left\{D_{0}^{v} / E_{1}, D_{1}^{v} / E_{1}\right\}$ is a 2-cycle cover of the contracted graph $\left(G^{v} / E_{1}, 2 \chi^{E_{2} \cup\left\{e_{v}\right\} \backslash\left\{e_{0}\right\}}\right)$. The arbitrary choice of $e_{0} \in E_{2}$ implies that exactly two of the three vertices in the contracted graph $G^{v} / E_{1}$ have odd degree, and that every edge in $E_{2}=E\left(G^{v} / E_{1}\right) \backslash\left\{e_{v}\right\}$ joins these two odd vertices. One easily sees that such a graph cannot exist (unless $G^{v} / E_{1}$ is disconnected, which clearly is not the case). This contradiction establishes that $V_{4}=\varnothing$.

Thus $G$ is cubic, the two components comprising $E_{1}$ are circuits in $G$, and every edge in the 1-factor $E_{2}$ has an endvertex in each of these circuits (such graphs are called $\sigma$-prisms in [Als]). Suppose that $G$ has a proper 3-edge coloring. Let $Z_{i}$ be the cycle obtained by deleting the $i$ th color class from $G, i=1,2,3$. Then $\left\{Z_{1}, Z_{2}, Z_{3}\right\}$ is a 3 cycle double cover of $G$, and hence $\left\{Z_{1} \triangle E_{1}, Z_{2} \triangle E_{1}, Z_{3} \triangle E_{1}\right\}$ is a cycle cover of $(G, p)$, a contradiction. Thus $G$ has no proper 3-edge coloring. By Lemma 3, the deletion of some edges $S \subseteq E_{2}$ yields a subdivision of Petersen's graph. Hence $\left(G, p-2 \chi^{S}\right)$ is a blistered $\left(P_{10}, p_{10}\right)$ such that $E_{1}$ induces exactly two disjoint circuits. By minimality of $p$, we must have $S=\varnothing$. Since $G$ is 3-edge-connected, we have $(G, p)=\left(P_{10}, p_{10}\right)$, and we have proved Lemma 2 .

\section{COMPLEXITY}

We do not know the complexity of deciding whether a general weighted graph has a circuit cover (we call this the circuit cover problem). The difficulty of the Shortest Circuit Cover Problem and the Cycle Double Cover Conjecture suggests that this problem is $\mathscr{N P}$-hard. Indeed, we do not even know whether the circuit cover problem belongs to either of the classes $\mathscr{N P}$ or co- $\mathscr{N P}$ (see [Gar] for definitions). It is conceivable that the number of distinct circuits needed in a circuit cover of $(G, p)$ grows linearly with $r:=\max \{p(e) \mid e \in E(G)\}$ rather than a polynomial in the input size $|E(G)| \log (r)$, hence the ambiguity of membership in $\mathscr{N P}$.

If we restrict the input to graphs with no $P_{10}$-minor, then the circuit cover problem belongs to the complexity class $\mathscr{P}$. (Incidentally, determining whether a graph has a $P_{10}$-minor can be done in polynomial time [Sey4].) Indeed, 
testing the admissibility of a weight vector $p$ requires only $|V(G)|$ parity checks and $|E(G)|$ applications of the Max-flow Min-cut algorithm, both of which are polynomial in $|E(G)| \log (r)$.

The following questions, however, warrant further investigation. Suppose that $G$ has no $P_{10}$-minor and $(G, p)$ is admissible.

(6.1) Does $(G, p)$ have a circuit cover where the number of distinct circuits is bounded by a polynomial in $|E(G)| \log (r)$ ?

(6.2) Is there a polynomial-time algorithm which will construct a circuit cover of $(G, p)$ ?

Of course (6.2) is stronger than (6.1). From the proof of Theorem 5 below, we shall see that $(6.1)$ holds true. In fact, if $(G, p)$ has a circuit cover and $G$ has no $P_{10}$-minor, then $(G, p)$ has a circuit cover using fewer than $2|E(G)|$ distinct circuits. The following is a partial answer to (6.2).

Theorem 5. Question (6.2) holds true if and only if there is a polynomial time algorithm for the following problem.

Input : $A$ bridgeless graph $H$ with maximum degree 4 and containing no $P_{10}$-minor, together with a cycle $Z$ in $H$.

Output: A circuit $C$ such that $\left(H, 2-\chi^{Z}-\chi^{C}\right)$ is admissible.

Proof. Suppose that (6.2) has a positive answer. By Proposition 6, the $\{1,2\}$ weighted graph $\left(H, 2-\chi^{Z}\right)$ is admissible, and hence has a circuit cover which can be constructed in polynomial time. Any one of the circuits in this cover can be used for $C$.

Conversely, let $(G, p)$ be an admissible weighted graph where $G$ has no $P_{10}$-minor, and let $\mathbf{O}$ denote an oracle which can solve (6.3) in polynomial time. We note that by applying oracle $\mathbf{O}$ repeatedly, one can obtain a circuit cover of $\left(H, 2-\chi^{Z}\right)$. A naive implementation (CirCov1, outlined below) based on the proof of Lemma 1 can find a circuit cover of $(G, p)$ using oracle $\mathbf{O}$. Unfortunately, CirCov1 is only pseudo-polynomial (see [Gar]) since the number of distinct circuits in the circuit cover $\mathbf{L}^{\prime}$ it produces can be proportional to $|E(G)| r$, where $r=\max \{p(e) \mid e \in E(G)\}$. We shall subsequently demonstrate, however, the existence of a strongly polynomial-time algorithm (CirCov2) which produces a pair $(\mathbf{L}, \mu)$ where $\mathbf{L}$ is a list of $t<2|E(G)|$ circuits in $G$, and where $\mu=\left(\mu_{1}, \ldots, \mu_{t}\right)$ is a corresponding multiplicity vector (whose entries are bounded by $r)$, such that $(\mathbf{L}, \mu)$ describes a circuit cover of $(G, p)$.

CirCov1 : Input : An admissible edge weighted $\operatorname{graph}(G, p)$ where $G$ has no $P_{10}$-minor.

Output : A circuit cover $\mathbf{L}^{\prime}$ of $(G, p)$.

1. Preprocessing: Delete edges of weight 0 . Reduce any nontrivial tight cut. Such a tight cut yields two admissible contracted graphs $\left(G_{1}, p_{1}\right),\left(G_{2}, p_{2}\right)$ (see Proposition 5) which are solved separately, then spliced appropriately at the tight cut. We assume from here that $(G, p)$ is 3-edge-connected, positive, admissible and that all tight cuts are trivial.

2. If $p=1$, then we exit with a circuit decomposition of the eulerian graph $G$. Otherwise let $e_{0}=x y$ be any edge of minimum weight subject to $e_{0}$ being a nonfollower (cf. (4.3)) having weight at least 2 . 
3. Call CirCov1 recursively to find a circuit cover $M$ of $\left(G, p-2 \chi^{e_{0}}\right)$.

4. As in the proof of Lemma 1, we find a shortest $(x, y)$-path in the auxiliary graph $G_{\mathbf{M}}$ and obtain a subset $\mathbf{M}^{\prime}=\left\{C_{1}, C_{2}, \ldots, C_{k}\right\} \subseteq \mathbf{M}$ having the form of Figure 2.

5. Use the Max-flow Min-cut algorithm on the auxiliary graph $H$ to find $p\left(e_{0}\right)$ closed trails $\mathbf{W}=\left\{W_{1}, W_{2}, \ldots, W_{r}\right\}$ as in (4.7), and use these to define, for each chain $\mathbf{Q} \subseteq \mathbf{M}^{\prime}$, the $\{0,1,2\}$-valued weight vector $q_{\mathbf{Q}} \leq p$.

6. For each chain $Q$ we apply oracle $\mathbf{O}$ repeatedly to find a circuit cover of $\left(G, q_{\mathbf{Q}}\right)$. This can be done since the support of $q_{\mathbf{Q}}$ is an admissible $\{1,2\}$ weighted subgraph of $G$ having maximum degree 4 and containing no $P_{10^{-}}$ minor. Finally, we combine these circuit covers as described at the end of the proof of Lemma 1 to obtain a circuit cover of $\left(G, \chi^{\mathbf{M}^{\prime}}+2 \chi^{e_{0}}\right)$. Adjoining the list of circuits $\mathbf{L}^{\prime} \backslash \mathbf{M}^{\prime}$ to this circuit cover gives the desired circuit cover of $(G, p)$. Exit.

Detecting nontrivial tight cuts in Step 1 requires $O(|E|)$ network flow calculations. Nonfollowers are easy to detect in Step 2 as all tight cuts are trivial here. Steps 4 through 6 also involve only network flow, shortest path, and parity check calculations and are easily seen to be polynomial in $|E|$ and the running time of oracle $\mathbf{O}$. Finally, the total number of invocations of CirCov1 is at most $p(G) / 2$ as the total weight of each successive graph is reduced by 2 .

A strongly polynomial algorithm for (6.2) can be obtained from CirCov1 by using a trick which first appeared in essence in a paper by Cook, Fonloupt, and Schrijver [Coo] regarding Hilbert bases. In the terminology of Hilbert bases, the main result of this paper can be stated as follows.

(6.4) The circuits of a graph form a Hilbert basis if and only if the graph has no $P_{10}$-minor.

The idea is to polynomially solve a linear program relaxation of the circuit cover problem for $(G, p)$, and to separate out any fractional part of the resulting solution. We then use CirCov1 to replace the (relatively small) fractional part with an integer solution.

Recall that $\mathrm{C}$ denotes the set of circuits in $G$. Let $M$ denote the circuitedge $\{0,1\}$-incidence matrix for $G$, let $\mathbf{1}$ denote the column vector of $|C|$ ones, and suppose that $p$ is a row vector.

CirCov2: Input: An admissible edge weighted graph $(G, p)$ where $G$ has no $P_{10}$ minor.

Output: A circuit cover $(\mathbf{L}, \mu)$ of $(G, p)$ where $\mathbf{L}$ is a list of at most $2|E(G)|-1$ circuits and $\mu$ is a multiplicity vector whose entries are bounded by $r=\max \{p[(e) \mid e \in E(G)\}$.

1. Find a basic feasible solution $\lambda=\left(\lambda_{C}\right)_{C \in C}$ to the following linear program:

$$
\begin{aligned}
& \max \lambda \mathbf{1} \\
& \lambda M=p \\
& \lambda \geq 0
\end{aligned}
$$

2. Let $\lfloor\lambda\rfloor:=\left(\left\lfloor\lambda_{C}\right\rfloor\right)_{C \in C}$ and $\{\lambda\}:=\lambda-\lfloor\lambda\rfloor$ be the integer and fractional parts of $\lambda$, and let $p^{\prime}:=\{\lambda\} M=p-[\lambda] M$. As $p^{\prime}$ is a nonnegative combination of circuits, $\left(G, p^{\prime}\right)$ is balanced. Furthermore, $\left(G, p^{\prime}\right)$ is eulerian since both $p$ and $\lfloor\lambda\rfloor M$ are. Thus $\left(G, p^{\prime}\right)$ is admissible. 
3. Call CirCovl with input $\left(G, p^{\prime}\right)$ to obtain a circuit cover $\mathbf{L}^{\prime}$ of $\left(G, p^{\prime}\right)$.

4. Adjoin $L^{\prime}$ to the circuit cover $(\Lambda,\lfloor\lambda\rfloor)$ of $\left(G, p-p^{\prime}\right)$, where $\Lambda:=\{C \in$ $\left.\mathbf{C} \mid\left\lfloor\lambda_{C}\right\rfloor>0\right\}$, and exit with the resulting circuit cover $(\mathbf{L}, \mu)$.

We bound the size of $L$ as follows. As $\lambda$ is a basic solution, $|\Lambda| \leq|E|$. Also, by maximality of $\lambda \mathbf{1}$ we have $\left|\mathbf{L}^{\prime}\right|+\lfloor\lambda\rfloor \mathbf{1} \leq \lambda \mathbf{1}=\lfloor\lambda\rfloor \mathbf{1}+\{\lambda\} \mathbf{1}$, so $\left|\mathbf{L}^{\prime}\right| \leq\{\lambda\} \mathbf{1}$. Since each of the nonzero entries in $\{\lambda\}$ is less than 1 we have $\{\lambda\} 1<|E|$, so $\left|\mathbf{L}^{\prime}\right| \leq|E|-1$. Thus $|\mathbf{L}| \leq|\Lambda|+\left|\mathbf{L}^{\prime}\right| \leq 2|E|-1$. Incidentally, this argument shows that $(6.1)$ is true as claimed above.

As $\max \left\{p^{\prime}(e) \mid e \in E(G)\right\} \leq\left|\mathrm{L}^{\prime}\right|<|E|$, Step 3 is strongly polynomial in the running time of oracle $\mathbf{O}$. It remains to show that Step 1 of CirCov 2 can be done in time bounded by a polynomial in $|E(G)| \log (r)$ despite the exponential number of variables $\lambda_{C}$. We give an indirect method which involves the dual linear program.

$$
\begin{aligned}
& \min p x \\
& M x \geq 1
\end{aligned}
$$

The separation problem for (6.6) is the following:

Given a rational weight vector $x$ on $E(G)$ either determine that $x$ satisfies $M x \geq 1$, or display a violated inequality (that is, a circuit in $G$ having total weight less than 1).

A deep theorem of Grötschel, Lovász and Schrijver (See Corollary 14.1g(v) of [Sch]) implies that a basic optimal solution to (6.5) can be found via the ellipsoid method in time polynomially bounded by $|E|$ and the input length of $w$ provided that

(i) the polyhedron $P:=\{x \mid M x \geq \mathbf{1}\}$ is full dimensional and pointed (see 8.3(6) in [Sch]), and

(ii) the separation problem for (6.6) can be solved in time polynomially bounded by $|E|$ and the input length of $x$.

That $P$ is full dimensional follows from the fact that any edge $e=s t$ in a 3edge-connected graph is a $\left\{0, \pm \frac{1}{2}\right\}$-linear combination of three cycles (consider two edge-disjoint $(s, t)$-paths in $G-e)$. To prove pointedness, suppose that weight vectors $x$ and $x^{\prime}$ are such that $x+\alpha x^{\prime} \in P$ for all rational scalars $\alpha$. Then we must have $M x^{\prime}=0$. As $P$ is full dimensional, the columns of $M$ are linearly independent so $x^{\prime}=\mathbf{0}$, and thus $P$ is pointed. To solve (ii) it suffices to check for each $e \in E(G)$ that $\left(G, x-\chi^{e_{0}}\right)$ has no negative-weight circuits or display one if one exists. This can be done using $|E(G)|$ calls to a shortest-path algorithm for undirected weighted graphs with no negative-weight circuits (e.g., Chapter 6.2 in [Law]). This completes the proof.

It is possible that a direct algorithm for solving (6.5) can be obtained using the proof of Seymour's "sums of circuits" result [(2.5) in Sey1], though we do not investigate this here. We do not know whether there exists a polynomial-time algorithm for (6.3), even when input is restricted to cubic graphs.

\section{ACKNOWLEDGMENT}

We thank A. Sebő for stimulating discussion regarding the complexity of circuit covers. 


\section{REFERENCES}

[Alo] N. Alon and M. Tarsi, Covering multigraphs by simple circuits, SIAM J. Algebraic Discrete Methods 6 (1985), 345-350.

[Als] B. R. Alspach and C-Q. Zhang, Cycle coverings of cubic graphs, Discrete Math. 111 (1993), 11-17.

[Arc] D. Archdeacon, Face colorings of embedded graphs, J. Graph Theory 8 (1984), 387-398.

[Ber] J. C. Bermond, B. Jackson, and F. Jaeger, Shortest coverings of graphs with cycles, J. Combin. Theory Ser. B 35 (1983), 297-308.

[Bon] J. A. Bondy, Small cycle double covers of graphs, Cycles and Rays (G. Hahn, G. Sabidussi, and R. Woodrow, eds.), Nato ASI Series C, vol. 301, Kluwer, Dordrecht and Boston, 1990, pp. 21-40.

[Cel] U. A. Celmins, On cubic graphs that do not have an edge 3-coloring, Ph.D. thesis, Department of Combinatorics and Optimization, University of Waterloo, Waterloo, Canada, 1984.

[Cat] P. Catlin, Double cycle covers and the Petersen graph, J. Graph Theory 13 (1989), 465-483.

[Coo] W. Cook, J. Fonloupt, and A. Schrijver, An integer analogue of Carathéodory's theorem, J. Combin. Theory Ser. B 40 (1986), 63-70.

[Edm] J. Edmonds and E. L. Johnson, Matching, Euler-tours, and the Chinese postman, Math. Programming 5 (1973), 88-124.

[Ell] M. N. Ellingham, Petersen subdivisions in some regular graphs, Congr. Numer. 44 (1984), 33-40.

[Fan1] G. Fan, Covering weighed graphs by even subgraphs, J. Combin. Theory Ser. B 49 (1990), 137-141.

[Fan2] __, Integer flows and cycle covers, J. Combin. Theory Ser. B 54 (1992), 113-122.

[Fle1] H. Fleischner, Eulersche Linien und Kreisüberdeckungen die vorgegebene Durchgange in den Kanten vermeiden, J. Combin. Theory Ser. B 2 (1980), 145-167.

[Fle2] H. Fleischner and A. Frank, On circuit decompositions of planar Eulerian graphs, J. Combin. Theory Ser. B 50 (1990), 245-253.

[For] L. R. Ford and D. R. Fulkerson, Flows in networks, Princeton Univ. Press, Princeton, NJ, 1962.

[Fu] X. Fu and L. A. Goddyn, Matroids with the circuit cover property (in preparation).

[Ful] D. R. Fulkerson, Blocking and antiblocking pairs of polyhedra, Mat. Programming 1 (1971), 168-194.

[Gar] M. R. Gary and D. S. Jonsonson, Computers and intractability: a guide to the theory of NP-completeness, Freeman, San Francisco, 1979.

[God1] L. A. Goddyn, Cycle double covers of graphs with Hamilton paths, J. Combin. Theory Ser. B 46 (1989), 253-254.

[God2] _ Cycle covers of graphs, Ph.D. thesis, Department of Combinatorics and Optimization, University of Waterloo, Waterloo, Canada, 1988.

[Gua] M. Guan and H. Fleischner, On the minimum weight cycle covering problem for planar graphs, Ars Combin. 20 (1985), 61-68.

[Hag] G. Haggard, Loops in duals, Amer. Math. Monthly 87 (1980), 654-656.

[Ita] A. Itai and M. Rodeh, Covering a graph by circuits, Automata, Languages, and Programming, Lecture Notes in Computer Science, no. 62, Springer, Berlin, 1978, pp. 289-299.

[Jac] B. Jackson, Shortest circuit covers and postman tours in graphs with a nowhere zero 4-flow, SIAM J. Comput. 19 (1990), 659-665.

[Jae1] F. Jaeger, Flows and generalized coloring theorems in graphs, J. Combin. Theory Ser. B 26 (1979), 205-216.

[Jae2] _ A survey of the cycle double cover conjecture, Cycles in Graphs (B. R. Alspach and C. D. Godsil, eds.), Ann. Discrete Math., vol. 27, North-Holland, Amsterdam, 1985, pp. $1-12$. 
[Jam1] U. Jamshy and M. Tarsi, Cycle covering of binary matroids, J. Combin. Theory Ser. B 46 (1989), 154-161.

[Jam2] U. Jamshy, A. Raspaud, and M. Tarsi, Short circuit covers for regular matroids with a nowhere zero 5-flow, J. Combin. Theory Ser. B 42 (1987), 354-357.

[Jam3] U. Jamshy and M. Tarsi, Short cycle covers and the cycle double cover conjecture, J. Combin. Theory Ser. B (submitted).

[Law] E. L. Lawler, Combinatorial optimization: networks and matroids, Holt, Rinehart and Winston, New York, 1976.

[Lit] C. Little and R. Ringeisen, On the strong graph embedding conjecture, Proc. Ninth Southeastern Conf. on Combinatorics, Graph Theory and Computing, Utilitas Math., Winnipeg, 1978, pp. 479-487.

[Mat] K. R. Matthews, On the Eulericity of a graph, J. Combin. Theory Ser. B 2 (1978), 143-148.

[Sch] A. Schrijver, Theory of linear and integer programming, Wiley, New York, 1986, pp. 310312.

[Sey1] P. D. Seymour, Sums of circuits, Graph Theory and Related Topics (J. A. Bondy and U. S. R. Murty, eds.), Academic Press, New York, 1979, pp. 341-355.

[Sey2] — Matroids and multicommodity flows, Europ. J. Combin. 2 (1981), 257-290.

[Sey3] _ Even circuits in planar graphs, J. Combin. Theory Ser. B 31 (1981), 327-338.

[Sey4] P. D. Seymour and N. Roberson, Graph Minors. XIII: The disjoint paths problem (submitted).

[Sze] G. Szekeres, Polyhedral decompositions of cubic graphs, J. Austral. Math. Soc. 8 (1973), 367-387.

[Tar1] M. Tarsi, Nowhere zero flows and circuit covering in regular matroids, J. Combin. Theory Ser. B 39 (1985), 346-352.

[Tar2] , Semi-duality and the cycle double cover conjecture, J. Combin. Theory Ser. B 41 (1986), 332-340.

[Tut] W. T. Tuttle, On the imbedding of linear graphs in surfaces, Proc. London Math. Soc. (2) 51 (1950), 474-483.

[Wel] D. Welsh, Matroid theory, Academic Press, San Francisco, 1976.

[You] D. H. Younger, Integer flows, J. Graph Theory 7 (1983), 349-357.

[Zel] B. Zelinka, On a problem of P. Vestergaard concerning circuits in graphs, Czechoslovak Math. J. 37 (1987), 318-319.

[Zha1] Cun-Quan Zhang, Minimal cycle coverings and integer flows, J. Graph Theory 14 (1990), 537-546.

[Zha2] - On compatible cycle decompositions of eulerian graphs, preprint.

[Zha3] _ On even cycle decompositions of eulerian graphs, preprint.

(Brian Alspach and Luis Goddyn) Department of Mathematics and Statistics, Simon Fraser University, Burnaby, British Columbia, Canada V5A 156

E-mail address: alspachøcs.sfu.ca

goddyn@math.sfu.ca

(Cun-Quan Zhang) Department of Mathematics, West Virginia University, MorganTOWN, WEST VIRGINIA 26506

E-mail address: cqzhang@wvnvm. wvnet. edu 